Resultados genéricos sobre entropia e dimensão de Hausdorff para difeomorfismos conservativos sobre superfícies

Thiago Aparecido Catalan 

SERVIÇO DE PÓS-GRADUAÇÃO DO ICMC-USP

Data de Depósito: 12 de dezembro de 2007

Assinatura:

\section{Resultados genéricos sobre entropia e dimensão de Hausdorff para difeomorfismos conservativos sobre superfícies ${ }^{1}$}

\section{Thiago Aparecido Catalan}

\section{Orientador: Prof. Dr. Ali Tahzibi}

Dissertação apresentada ao Instituto de Ciências Matemáticas e de Computação - ICMC/USP, como parte dos requisitos para obtenção do título de Mestre em Matemática.

\section{USP - São Carlos}

\section{Dezembro/2007}

\footnotetext{
${ }^{1}$ Projeto financiado pela FAPESP
} 

Aos meus pais, Walter e Angela, e meu irmão, Cristiano. 



\section{Agradecimentos}

Primeiramente gostaria de agradecer a Deus, pois sem sua benção nada seria possível.

Aos meus Pais, Walter e Angela, serei eternamente grato. Obrigado pela confiança, pelos conselhos, e ensinamentos. Tenho em vocês a minha força e a minha base. Acredito que família é um item fundamental na vida de qualquer pessoa, e vocês me proporcionaram um ambiente familiar simplesmente espetáculo. Muito obrigado mesmo. É bom de mais ser filho de vocês. Ah, acho que as chineladas tiveram um bom efeito.

Agradeço ao meu irmão Cristiano, ou melhor, Ticé ${ }^{2}$ pela companhia durante todos estes anos. Pelas partidas de futebol no quintal, as quedas de braço, as quais eu sempre ganhava, e não poderia deixar de lembrar das belas brigas na infância, a Maria que o diga! Você com certeza é o melhor irmão do mundo, obrigado.

Sei que esta frase é batida, mas realmente faltam-me palavras para agradecer a minha namorada Fernanda. Ficaria um tempo não enumerável escrevendo se tentasse descrever o que sinto por você, e o quanto sou grato. Muito obrigado pelo companheirismo, amizade, amor, lealdade, e carinho. Obrigado por sempre estar presente, pelas tantas horas de ônibus até São Carlos (será que foram muitas?), pelos belos finais de semana, etc. Saiba que em você tenho a razão do meu viver.

Aos amigos que fiz no IBILCE, agradeço a todos, mas não poderia deixar de citar o pessoal da minha turma do Bacharel: Grasi, Vina, Agnaldo, Ligia, Carol e Dani. Obrigado também a todos os professores em especial o pessoal de Sistemas Dinâmicos não só pela formação Matemática, mas também pela amizade. Aproveitando o momento, agradeço ao Prof. Dr. Vanderlei pela orientação durante minha iniciação científica, a qual foi fundamental na minha base matemática, pela amizade, conselhos, e oportunidades apresentadas.

Aos companheiros de TranQra, Morera e Jaca, pelas conversas, baladas, e grandes partidas de sinuca, muito obrigado. Vocês são amigos que terei sempre comigo, amigos do peito, amigos de TranQra.

Agradeço aos companheiros da sala 4-232, a mais famosa sala do ICMC: Pimenta, Juliano, Patrícia, Luciene e o nosso representante Luiz Roberto Hartmann Júnior, um grande amigo 
que sempre atendeu aos meus chamados para solucionar algum problema computacional sem medir esforços ( será?! ). Aos amigos que fiz no ICMC: Juliano (Charanga), Marquinhos, Micena, Março Feniu, Baiano, Tatimi, Wescley, Giu, Castilho, Duzinho, Leitão, Thaís (a mais legal!!!), e muitos outros.

Aos companheiros de Morada do Escorpião: Claudinei, Kenji, Mateus e Fausto, agradeço pelas boas risadas, baladas, além dos atos maldosos. Ah, será que alguém tem chocolate?

Ao Prof. Dr. Ali Tahzibi agradeço pela orientação, dedicação, e paciência. Obrigado por me encorajar em horas difíceis, e pelos conhecimentos ensinados.

Aos funcionários do ICMC, o meu muito obrigado.

Por fim, mas não menos importante, agradeço a FAPESP pelo fomento. 


\section{Resumo}

Apresentamos duas propriedades genéricas para difeomorfismos conservativos de classe $C^{1}$ sobre uma superfície compacta de dimensão dois. Obtemos uma limitação inferior para entropia topológica de difeomorfismos genéricos, e mostramos que tais difeomorfismos sempre possuem conjuntos invariantes fechados com órbitas densas e dimensão de Hausdorff dois. 

We present two generic properties of $C^{1}$ area preserving diffeomorphisms of a two dimensional compact oriented surface. We obtain a lower bound for the topological entropy of a generic diffeomorphisms, and we show that such a diffeomorphism always has closed invariant sets with dense orbits and Hausdorff dimension two. 



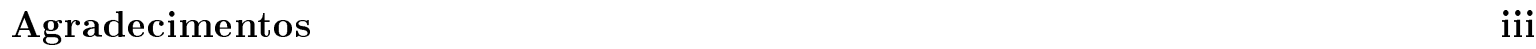

Resumo $\quad$ v

Abstract $\quad$ vii

$\begin{array}{ll}\text { Introdução } & 1\end{array}$

1 Dinâmica Hiperbólica $\quad 3$

1.1 Propriedades Topológicas . . . . . . . . . . . . . . . . 3

1.1.1 Entropia Topológica . . . . . . . . . . . . . . . . 8

1.2 Propriedades Ergódicas _. . . . . . . . . . . . . . . . . . . . . 10

1.2.1 Entropia Métrica . . . . . . . . . . . . . . . . . . . . . . 10

1.2 .2 Estado de Equilíbrio e a função $\phi^{u} \ldots \ldots$. . . . . . . . . . . . . 12

2 Preliminares de Perturbação $\quad 15$

2.1 Função Geradora . . . . . . . . . . . . . . . . . . . . . . . . . 15

2.2 Lema de Perturbação . . . . . . . . . . . . . . . . . . . . . . . . . . 20

3 Resultado Principal $\quad 23$

3.1 Dimensão de Hausdorff e o funcional $s(.) \ldots \ldots \ldots \ldots \ldots \ldots$

3.2 Propriedades genéricas para $\operatorname{Diff}_{\omega}^{1}(M) \ldots \ldots \ldots \ldots$. . . . . . . . . . . . . . . . . . . . . . .

3.3 Difeomorfismos de Anosov . . . . . . . . . . . . . . . . . . . . . 42

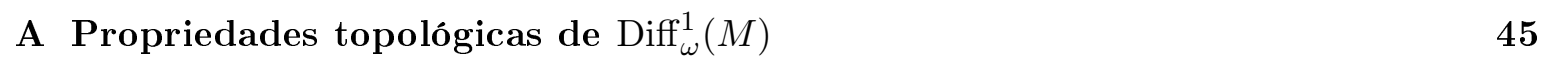

A.1 $\operatorname{Diff}_{\omega}^{1}(M)$ é um espaço de Baire . . . . . . . . . . . . . . . . 45

$\begin{array}{ll}\text { Referências Bibliográficas } & 49\end{array}$ 
Tabela de Símbolos e Abreviações

Índice Remissivo 
A entropia topológica é um invariante para difeomorfismos muito útil no que se refere a "medir"a caoticidade de um Sistema Dinâmico. No entanto, é muito difícil de se calcular a entropia de um sistema. Sendo assim, tornam-se interessantes estudos que visam encontrar uma limitação inferior para entropia. Continuando nesta procura por meios diferentes de se estimar a entropia, olhamos para os pontos homoclínicos, introduzidos por Henry Poincaré no estudo do problema restrito de três corpos. Sabemos que a existência de um ponto homoclínico transversal é fonte de um conjunto hiperbólico, para o qual a entropia é positiva. E portanto, a entropia do sistema também é positiva. Com isto, aumenta o interesse de se saber o quão "gorda"são as classes homoclínicas de um sistema. Uma boa forma de se medir isto seria usando dimensão de Hausdorff.

Seja $M$ uma variedade orientável, compacta, conexa, de dimensão dois. Neste trabalho apresentamos duas propriedades genéricas em $\operatorname{Diff}_{\omega}^{1}(M)$, isto é, sobre o conjunto dos difeomorfismos de classe $C^{1}$, que preservam área, sobre $M$. Obtemos uma limitação inferior para entropia topológica de difeomorfismos genéricos, e mostramos que tais difeomorfismos sempre possuem conjuntos invariantes fechados com órbitas densas e dimensão de Hausdorff dois, [1]. É claro que esta limitação inferior, para que seja interessante e útil, tem que ser algo calculável. No caso, nossa limitação inferior é o funcional $s($.$) , definido como sendo o supremo$ sobre os expoentes de Liapunov dos pontos periódicos hiperbólicos. Também, como estamos falando de propriedades genéricas, precisamos que $s($.$) tenha propriedades topológicas boas.$ De fato, temos que $s($.$) é semi-contínuo superior. Por fim, denotando por H(p, f)$, a classe homoclínica de um ponto periódico hiperbólico $p$, para $f$, o resultado principal deste trabalho é o seguinte: 
Teorema 1. Existe um conjunto residual $\mathcal{B} \subset \operatorname{Diff}_{\omega}^{1}(M)$ tal que se $f$ está em $\mathcal{B}$, então cada conjunto $\overline{H(p, f)}$ tem dimensão de Hausdorff dois. Mais ainda, se $f$ está em $\mathcal{B}$ e não é Anosov, então

$$
h(f) \geq s(f) .
$$

Para difeomorfismos de Anosov, usando técnicas apresentadas por Bowen em [6], mostramos que a equação (1) falha num conjunto aberto e denso. Também, no caso Anosov em dimensão dois, usando [4], temos que toda classe homoclínica é densa sobre a variedade $M$. Logo, a primeira parte do Teorema 1 é trivialmente satisfeita. Sendo assim, para provarmos o Teorema 1, precisamos apenas nos preocupar com os difeomorfismos não Anosov.

Agora, para difeomorfismos não Anosov, Newhouse mostrou em [2], que no caso simplético a única patologia para um difeomorfismo não ser anosov é ser aproximado por tangências ( veja conjectura de Palis). Isto é, no complementar dos difeomorfismos hiperbólicos existe um conjunto denso de difeomorfismos que exibem tangências homoclínicas. Usando as mesmas técnicas que Newhouse, juntamente com um resultado genérico de Takens, o de que as classes homoclínicas são densas nas variedades estáveis e instáveis, mostramos que todo ponto periódico hiperbólico de um difeomorfismo não anosov, exibe uma tangência homoclínica, a menos de uma perturbação.

A partir disto, podemos fazer perturbações numa vizinhança desta tangência, conseguindo gerar um conjunto "gordo"dentro da classe homoclínica, possuindo dimensão de Hausdorff máxima, o qual utilizamos, também, para mostrar a desigualdade (1).

Notemos que o resultado principal deste trabalho é válido para variedades com dimensão dois. Um resultado recente de Bonatti e Crovisier [13], mostra que $\overline{H(p, f)}=M$ para todo ponto periódico $p$ de um difeomorfismo conservativo $f$, para $M$ de dimensão qualquer. $\mathrm{O}$ que generaliza a primeira parte do Teorema 1. Pensando nisto, podemos nos perguntar se é possível generalizar a segunda parte do Teorema 1. 


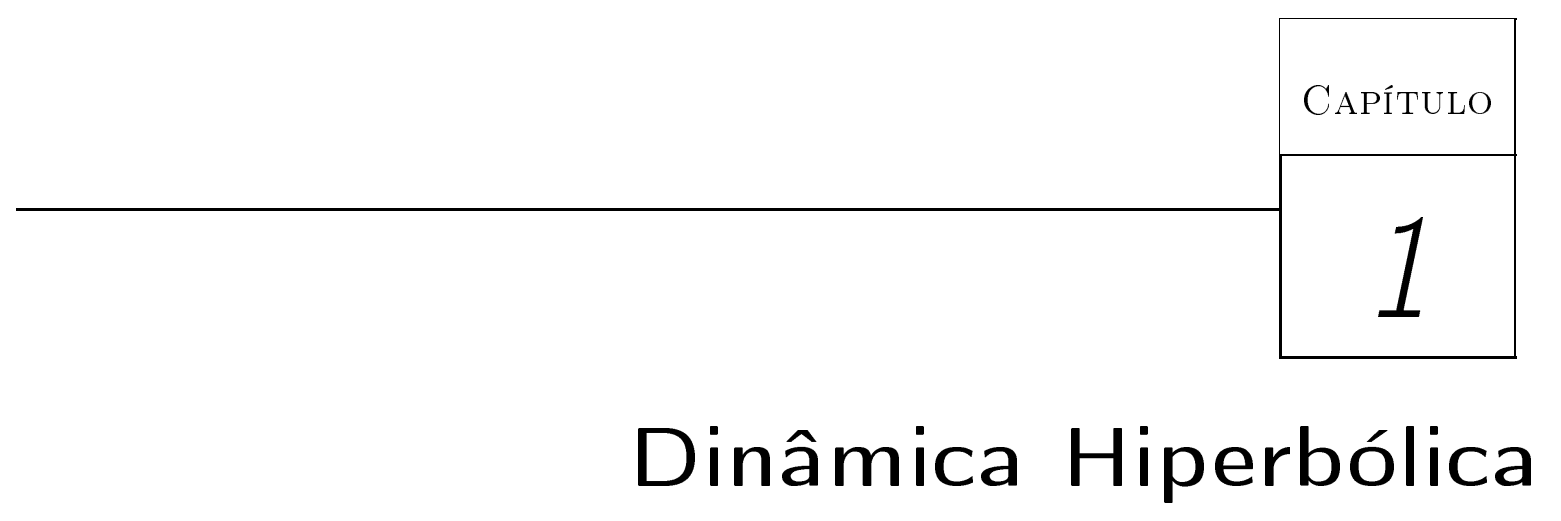

Neste capítulo vamos introduzir alguns dos resultados centrais da teoria de Sistemas Dinâmicos. Estes resultados podem ser divididos em dois grandes conjuntos, os que expressam as propriedades topológicas dos sistemas, e aqueles que expressam as propriedades ergódicas.

\subsection{Propriedades Topológicas}

Todos os resultados apresentados nesta seção, juntamente com suas respectivas demonstrações, podem ser encontrados em [5].

Vamos considerar $M$ uma variedade, $C^{\infty}$, compacta, conexa e orientável. Denotamos por $\operatorname{Diff}^{1}(M)$ o conjunto dos difeomorfismos de classe $C^{1}$ sobre $M$.

Nesta seção, quando não estiver especificado, consideramos $f \mathrm{em} \mathrm{Diff}^{1}(M)$.

Definição 1.1.1. Um ponto $p$ é um ponto periódico de período $n$ para $f$, se $f^{n}(p)=p$, e $f^{j}(p) \neq p$ para $0<j<n$. Se $p$ possui período um, então ele é chamado de ponto fixo. Denotamos por $o(p)$ a órbita de um ponto periódico $p$, e por $\tau(p)$ o período do ponto $p$.

Definição 1.1.2. Um ponto periódico $p$ é chamado hiperbólico se a aplicação linear $D f^{\tau(p)}(p)$ possui autovalores com valor absoluto diferente de um. Onde $D f^{\tau(p)}(p)$ é a derivada de $f^{\tau(p)}$ no ponto $p$.

Definição 1.1.3. Um ponto periódico $p$ é chamado elíptico se $D f^{\tau(p)}(p)$ possui autovalores não reais com norma um. 
Podemos restringir um pouco mais a definição acima.

Definição 1.1.4. Dizemos que um ponto periódico $p$ para $f$, é $k$-elíptico, $1 \leq k \leq \frac{\operatorname{dim} M}{2}$, se $D f^{\tau(p)}(p)$ possui $2 k$ autovalores não reais de norma um, e os outros autovalores possuem norma diferente de um.

Observemos que no caso de uma variedade $M$ de dimensão 2, temos uma equivalência na definição de pontos 1-elípticos e pontos elípticos.

Definição 1.1.5. Um conjunto invariante $\Lambda$ possui uma estrutura hiperbólica para um difeomorfismo $f$, se:

(i) Em cada ponto $p \in \Lambda$ o espaço tangente de $M$ é fatorado numa soma direta de $\mathbb{E}_{p}^{u}$ e $\mathbb{E}_{p}^{s}$, $T_{p} M=\mathbb{E}_{p}^{u} \oplus \mathbb{E}_{p}^{s}$

(ii) Esta fatoração é invariante pela derivada $D f$. Isto é,

$$
D f(p)\left(\mathbb{E}_{p}^{u}\right)=\mathbb{E}_{f(p)}^{u} \text { e } D f(p)\left(\mathbb{E}_{p}^{s}\right)=\mathbb{E}_{f(p)}^{s} .
$$

(iii) Existem $0<\lambda<1$ e $C \geq 1$, independentes de $p$, tal que para todo $n \geq 0$,

$$
\begin{gathered}
\left|D f^{n}(p)\left(v^{s}\right)\right| \leq C \lambda^{n}\left|v^{s}\right| \text { para } v^{s} \in \mathbb{E}_{p}^{s}, \text { e } \\
\left|D f^{-n}(p)\left(v^{u}\right)\right| \leq C \lambda^{n}\left|v^{u}\right| \text { para } v^{u} \in \mathbb{E}_{p}^{u} .
\end{gathered}
$$

Se um conjunto invariante $\Lambda$ possui uma estrutura hiperbólica para $f$, dizemos que $\Lambda$ é um conjunto hiperbólico.

Dado um conjunto hiperbólico $\Lambda$, por definição, segue que $\mathbb{E}_{p}^{u}$ e $\mathbb{E}_{p}^{s}$ variam continuamente com $p$. Também, se $m$ é um inteiro positivo tal que $\rho=C \lambda^{m}<1$, então

$$
\begin{aligned}
\left|D f^{m}(p)\left(v^{s}\right)\right| & \leq \rho\left|v^{s}\right| \text { para } v^{s} \in \mathbb{E}_{p}^{s}, \text { e } \\
\left|D f^{-m}(p)\left(v^{u}\right)\right| & \leq \rho\left|v^{u}\right| \text { para } v^{u} \in \mathbb{E}_{p}^{u} .
\end{aligned}
$$

Daí, $D f^{m}(p) \mid \mathbb{E}_{p}^{s}$ é uma contração e $D f^{m}(p) \mid \mathbb{E}_{p}^{u}$ é uma expansão. Por isto, diremos que $\mathbb{E}_{p}^{s}$ é a direção estável, e $\mathbb{E}_{p}^{u}$ a direção instável de $f$ no ponto $p$. Notemos que a constante $C$ determina o número de iterados de $f$ necessários, para que os vetores comecem a contrair (respectivamente, expandir) no subfibrado $\mathbb{E}_{p}^{s}$ (respectivamente, $E_{p}^{u}$ ).

Exemplo 1.1.6. Suponhamos $\Lambda$ um conjunto hiperbólico para $f^{N}$. Assim, por definição, para cada $x \in \Lambda$,

$$
T_{x} \Lambda=\mathbb{E}_{x}^{u} \oplus \mathbb{E}_{x}^{s}
$$

e esta decomposição varia continuamente. Além disto, existem constantes $C>0$ e $0<\lambda<1$, tal que

$$
\begin{gathered}
\left|D f^{n N}(p)\left(v^{s}\right)\right| \leq C \lambda^{n}\left|v^{s}\right| \text { para } v^{s} \in \mathbb{E}_{p}^{s}, \text { e } \\
\left|D f^{-n N}(p)\left(v^{u}\right)\right| \leq C \lambda^{n}\left|v^{u}\right| \text { para } v^{u} \in \mathbb{E}_{p}^{u} .
\end{gathered}
$$


Consideremos

$$
\bar{\Lambda}=\bigcup_{j=0}^{N} f^{j}(\Lambda),
$$

o conjunto invariante para $f$, construído naturalmente a partir de $\Lambda$. Os candidatos a direção instável e estável do conjunto $\bar{\Lambda}$, são os iterados de $\mathbb{E}^{u}$ e $\mathbb{E}^{s}$ por $f$. Ou seja,

$$
\mathbb{E}_{f^{j}(x)}^{i}=D f^{j}\left(\mathbb{E}^{i}(x)\right) \text {, para } i=u, s \text { e } j=1, \ldots, n-1 .
$$

As primeiras duas propriedades $(i)$ e $(i i)$, da Definição 1.1.5, são satisfeitas por construção. Agora, dado $n \geq 0$, seja $k \in \mathbb{N}$ e $0 \leq r<N$ tal que $n=k N+r$. Tomando $C_{1}=$ $C \lambda^{-N} \max \left\{\left\|D f^{i}\right\|\right.$, para $\left.0 \leq j<N\right\}$, e $\lambda_{1}=\lambda^{\frac{1}{N}}$, temos que:

$$
\begin{aligned}
\left|D f_{x}^{n}\left(v^{s}\right)\right| & \leq\left\|D f^{r}\right\|\left|D f_{x}^{k N}\left(v^{s}\right)\right| \\
& \leq C_{1} \lambda_{1}^{n}\left|v^{s}\right|
\end{aligned}
$$

para todo $x \in \bar{\Lambda}$, e $v^{s} \in \mathbb{E}_{x}^{s}$. Com um processo análogo para $x \in \bar{\Lambda}$ e $v^{u} \in \mathbb{E}_{x}^{u}$, podemos concluir que $\bar{\Lambda}$ é hiperbólico.

Exemplo 1.1.7. Dado um ponto periódico $p$ para um difeomorfismo $f$, temos que $o(p)$ é um conjunto invariante para $f$. Assim, dizer que $p$ é hiperbólico é equivalente a dizer que o conjunto $o(p)$ é hiperbólico.

Definição 1.1.8. Dado um difeomorfismo $f \in \operatorname{Diff}^{1}(M)$, dizemos que $f$ é um difeomorfismo de Anosov se a variedade $M$ é um conjunto hiperbólico.

Definição 1.1.9. Duas funções $f: \Lambda_{1} \rightarrow \Lambda_{1}$ e $g: \Lambda_{2} \rightarrow \Lambda_{2}$ são topologicamente conjugadas, se existe um homeomorfismo $h: \Lambda_{1} \rightarrow \Lambda_{2}$, tal que $g(h(x))=h(f(x))$.

Definição 1.1.10. Para uma aplicação $f: M \rightarrow M$, um ponto $p$ é chamado não errante se para toda vizinhança $U$ de $p$, existe um inteiro $n>0$ tal que $f^{n}(U) \cap U \neq \emptyset$. Isto é, existe um ponto $q \in U$ tal que $f^{n}(q) \in U$. O conjunto de todos os pontos não errantes para $f$, é chamado de conjunto não errante e é denotado por $\Omega(f)$.

Definição 1.1.11. Um difeomorfismo $f \in \operatorname{Diff}^{1}(M)$ é chamado de Axioma $A$ se o conjunto $\Omega(f)$ é hiperbólico e $\overline{\operatorname{Per}(f)}=\Omega(f)$.

Teorema 1.1.12 (Teorema de Hartman-Grobman). Seja $f \in \operatorname{Diff}^{1}(M)$, e p um ponto fixo hiperbólico. Então, existem vizinhanças $U$ de $p, V$ de 0 , e um homeomorfismo $h: V \rightarrow U$, tal que $f(h(x))=h(A x)$ para todo $x \in V$, onde $A=D f(p)$. Em outras palavras, $f \dot{e}$ topologicamente conjugado a sua derivada numa vizinhança de $p$.

Observação 1.1.13. Em dimensão dois, Hartman provou que próximo de um ponto fixo hiperbólico, qualquer difeomorfismo $C^{2}$ é $C^{1}$ linearizável. Ou seja, é $C^{1}$ conjugado com a sua derivada, [12]. 
Teorema 1.1.14 (Teorema da Variedade Estável). Seja $f \in \operatorname{Diff}^{k}(M)$. Seja $\Lambda$ um conjunto hiperbólico para $f$, com constantes hiperbólicas $0<\lambda<1$ e $C \geq 1$. Então, existe $\varepsilon>0$ tal que para cada $p \in \Lambda$, existem dois discos $C^{k}$ em $M, W_{\varepsilon}^{s}(p, f)$ e $W_{\varepsilon}^{u}(p, f)$, que são tangentes a $\mathbb{E}_{p}^{s}$ e $\mathbb{E}_{p}^{u}$, respectivamente. Em ordem, para considerarmos esses discos como gráficos de funções, identificamos uma vizinhança de cada ponto p com $\mathbb{E}_{p}^{u}(\varepsilon) \times \mathbb{E}_{p}^{s}(\varepsilon)$. (Sobre variedades, esta identificação pode ser feita por coordenadas locais.) Usando esta identificação, para $i=s, u$ e $j=\{s, u\} \backslash\{i\}, W_{\varepsilon}^{i}(p, f)$ é gráfico de uma função $C^{k}, \sigma_{p}^{i}: \mathbb{E}_{p}^{i}(\varepsilon) \rightarrow \mathbb{E}_{p}^{j}(\varepsilon)$, com $\sigma_{p}^{i}\left(0_{p}\right)=0_{p}$ e $D\left(\sigma_{p}^{i}\right)_{0}=0$ :

$$
W_{\varepsilon}^{i}(p, f)=\left\{\left(y, \sigma_{p}^{i}(y)\right): y \in \mathbb{E}_{p}^{i}(\varepsilon)\right\} .
$$

Também, a função $\sigma_{p}^{i}$ e suas $k$ primeiras derivadas variam continuamente com $p$.

Mais ainda, identificando uma vizinhança de $p$ com $\mathbb{E}_{p}^{u}(\varepsilon) \times \mathbb{E}_{p}^{s}(\varepsilon)$ e tomando $\lambda<\lambda^{\prime}<1$, para $\varepsilon>0$ pequeno o suficiente,

$$
W_{\varepsilon}^{s}(p, f)=\left\{q \in \mathbb{E}_{p}^{u}(\varepsilon) \times \mathbb{E}_{p}^{s}(\varepsilon) ; d\left(f^{j}(q), f^{j}(p)\right) \leq C\left(\lambda^{\prime}\right)^{j} d(q, p) \text { para todo } j \geq 0\right\} .
$$

Similarmente,

$$
W_{\varepsilon}^{u}(p, f)=\left\{q \in \mathbb{E}_{p}^{u}(\varepsilon) \times \mathbb{E}_{p}^{s}(\varepsilon) ; d\left(f^{-j}(q), f^{-j}(p)\right) \leq C\left(\lambda^{\prime}\right)^{j} d(q, p) \text { para todo } j \geq 0\right\} .
$$

Depois de obtido, localmente, as variedades estável e instável, pelo Teorema 1.1.14, podemos determinar estas variedades globalmente como segue:

$$
\begin{aligned}
& W^{s}(p, f)=\left\{q \in M ; d\left(f^{j}(q), f^{j}(p)\right) \rightarrow 0, \text { quando } j \rightarrow \infty\right\} \\
& =\bigcup_{n \geq 0} f^{-n}\left(W_{\varepsilon}^{s}\left(f^{n}(p), f\right)\right), \\
& \text { e } \\
& W^{u}(p, f)=\left\{q \in M ; d\left(f^{-j}(q), f^{-j}(p)\right) \rightarrow 0, \text { quando } j \rightarrow \infty\right\} \\
& =\bigcup_{n \geq 0} f^{n}\left(W_{\varepsilon}^{u}\left(f^{-n}(p), f\right)\right) .
\end{aligned}
$$

Teorema 1.1.15 (Lambda Lema). Seja $p$ um ponto fixo hiperbólico para um difeomorfismo $f$ de classe $C^{k}$. Seja $r>0$, pequeno o suficiente, tal que numa vizinhança de $p$ dada por $\{p\}+\left(\mathbb{E}^{u}(r) \times \mathbb{E}^{s}(r)\right)$, as estimativas hiperbólicas dadas pelo Teorema da Variedade Estável 1.1.14 sejam válidas. Seja $D^{u}$ um disco topológico de mesma dimensão que $\mathbb{E}^{u}$ e tal que $D^{u}$ seja trasversal a $W_{r}^{s}(p, f)$. Seja $D_{1}^{u}=f\left(D^{u}\right) \cap\left(\mathbb{E}^{u}(r) \times \mathbb{E}^{s}(r)\right)$ e $D_{n+1}^{u}=f\left(D_{n}^{u}\right) \cap\left(\mathbb{E}^{u}(r) \times\right.$ $\left.\mathbb{E}^{s}(r)\right)$. Então, $D_{n}^{u}$ converge a $W_{r}^{u}(p, f)$ na topologia $C^{k}$.

Definição 1.1.16. Um conjunto hiperbólico $\Lambda$ é dito básico hiperbólico se $f \mid \Lambda$ possui uma órbita densa, e existe uma vizinhança compacta $U$ de $\Lambda$ tal que

$$
\bigcap_{-\infty<n<\infty} f^{n}(U)=\Lambda
$$

Chamamos $U$ de vizinhança isoladora. 
Observação 1.1.17. Dado um ponto $p$ periódico hiperbólico de período $n$ para $f$, podemos olhar para ele como um ponto fixo do difeomorfismo $f^{n}$. Pelo Teorema de Hartman-Grobman 1.1.12, podemos tomar uma vizinhança $U^{\prime}$ de $p$, suficientemente pequena, tal que

$$
\{p\}=\bigcap_{-\infty<j<\infty} f^{n j}\left(U^{\prime}\right)
$$

Assim, temos que $U=U^{\prime} \cup f\left(U^{\prime}\right) \cup \ldots \cup f^{n-1}\left(U^{\prime}\right)$ é uma vizinhança isoladora para $o(p)$. Logo, $o(p)$ é um conjunto básico hiperbólico..

Teorema 1.1.18 (Estabilidade de um Conjunto Básico Hiperbólico ). Seja $\Lambda_{f}$ um conjunto básico hiperbólico para $f \in \operatorname{Diff}^{1}(M)$, com vizinhança isoladora $U$. Existe $\varepsilon>0$, tal que se $g \in \operatorname{Diff}^{1}(M)$ e está $\varepsilon-C^{1}$ próximo de $f$, então g possui uma estrutura hiperbólica sobre

$$
\Lambda_{g}=\bigcap_{-\infty<j<\infty} g^{j}(U)
$$

e ainda, existe um homeomorfismo $h: \Lambda_{g} \rightarrow \Lambda_{f}$ que dá uma conjugação topológica entre $g \mid \Lambda_{g}$ e $f \mid \Lambda_{f}$.

Durante o texto, usaremos muitas vezes o termo continuação de um conjunto hiperbólico ou de um ponto periódico hiperbólico, estando implícito o Teorema 1.1.18.

Definição 1.1.19. Seja $p$ um ponto periódico hiperbólico de período $n$ para um difeomorfismo f. Seja

$$
\begin{aligned}
& W^{i}(o(p))=\bigcup_{j=0}^{n-1} W^{i}\left(f^{j}(p)\right), \mathrm{e} \\
& \hat{W}^{i}(o(p))=W^{i}(o(p)) \backslash o(p),
\end{aligned}
$$

para $i=s, u$. Um ponto $q \in \hat{W}^{s}(o(p)) \cap \hat{W}^{u}(o(p))$ é chamado de ponto homoclínico para $p$. Um ponto $q$ é chamado de ponto homoclinico transversal caso as variedades $\hat{W}^{s}(o(p))$ e $\hat{W}^{u}(o(p))$ tenham uma interseção transversal não vazia em $q$. Denotaremos por $H(p, f)$ o conjunto dos pontos homoclínicos transversais de $p$, com respeito a $f$.

O teorema seguinte, devido a Smale, mostra a existência de pontos periódicos tão perto, quanto se queira, de um ponto homoclínico transversal.

Teorema 1.1.20. Suponhamos que q seja um ponto homoclinico tranversal para um ponto periódico hiperbólico $p$ de um defeomorfismo $f$. Para cada vizinhança $U$ de $\{p, q\}$, existe um inteiro positivo $n$ tal que $f^{n}$ possui um conjunto hiperbólico $\Lambda \subset U$, com $p, q \in \Lambda$, e $\Lambda \subset \overline{\operatorname{Per}(f)}$. 


\subsubsection{Entropia Topológica}

Introduzimos agora um conceito muito importante, conhecido por Entropia Topológica. Podemos olhar para este, como uma maneira de "calcular"ou "medir"o quão caótico um sistema é. Dizemos isto, pois, como veremos ele é determinado pela taxa de crescimento do número de órbitas distintas do sistema.

Não só a definição de entropia topológica, como também outras propriedades, além das que aqui já estão, podem ser encontrados em [5].

Para definirmos entropia topológica, precisamos apenas que $X$ seja um um espaço métrico. É claro, que as propriedades interessantes acontecem quando $X$ também é compacto

Definição 1.1.21. Seja $f: X \rightarrow X$ uma aplicação contínua sobre $X$ com métrica $d$. Um conjunto $S \subset X$ é chamado $(n, \varepsilon)$-separado se dado $x \neq y$ em $S$, existe $0 \leq j<n$ tal que

$$
d\left(f^{j}(x), f^{j}(y)\right)>\varepsilon
$$

Definindo

$$
d_{n, f}(x, y)=\sup _{0 \leq j<n} d\left(f^{j}(x), f^{j}(y)\right)
$$

poderíamos definir $S \subset X(n, \varepsilon)$-separado, se $d_{n, f}(x, y)>\varepsilon$, para todo $x \neq y \in S$.

O número de órbitas $\varepsilon$-distintas de comprimento $n$, é definido por

$$
r(n, \varepsilon, f)=\max \{\sharp(S): S \subset X \text { é }(n, \varepsilon)-\text { separado para } f\} \text {. }
$$

Queremos calcular a taxa de crescimento de $r(n, \varepsilon, f)$ quando $n \rightarrow \infty$. Então, definimos

$$
h(\varepsilon, f)=\limsup _{n \rightarrow \infty} \frac{\log r(n, \varepsilon, f)}{n}
$$

Notemos que $r(n, \varepsilon, f) \geq 1$ para qualquer par $(n, \varepsilon)$, logo $0 \leq h(\varepsilon, f) \leq \infty$. Também, pode ser visto facilmente, que $h\left(\varepsilon_{1}, f\right) \leq h\left(\varepsilon_{2}, f\right)$ para $\varepsilon_{2}<\varepsilon_{1}$.

Finalmente, definimos a entropia topológica de $f$ como:

$$
h(f)=\lim _{\varepsilon \rightarrow 0^{+}} h(\varepsilon, f) .
$$

A proposição seguinte é uma propriedade muito útil de entropia, a qual vamos usar mais a frente.

Proposição 1.1.22. Seja $X$ um espaço métrico compacto, e $f: X \rightarrow X$ uma aplicação contínua. Então, dado $k$ um inteiro, com $k \geq 1$, a entropia de $f^{k}$ é igual a $k$ vezes a entropia de f. Isto é,

$$
h\left(f^{k}\right)=k h(f) .
$$


Demonstração. Inicialmente temos que

$$
\left\{f^{k i}(y): 0 \leq i<n\right\} \subset\left\{f^{i}(y): 0 \leq i<n k\right\} \text {, para todo } y \in X .
$$

Assim, se $d_{n, f^{k}}(x, y)>\delta$, então $d_{n k, f}(x, y)>\delta$, para todo $x, y \in X$. Isto implica, que se $S$ é $(n, \delta)$-separado para $f^{k}$, então $S$ é $(n k, \delta)$-separado para $f$. E assim,

$$
r\left(n, \delta, f^{k}\right) \leq r(n k, \delta, f) .
$$

Como $X$ é compacto, temos que $f$ é uniformemente contínua, e assim, dado $\varepsilon>0$, existe $\delta_{\varepsilon}>0$ tal que

$$
d(x, y)<\delta_{\varepsilon} \Rightarrow d\left(f^{j}(x), f^{j}(y)\right)<\varepsilon, 0 \leq j<k .
$$

Agora, se $S$ é $(n k, \varepsilon)$-separado para $f$, então existem $m$ e $j$ tal que $d\left(f^{j}(x), f^{j}(y)\right)>\varepsilon$ para $k(m-1) \leq j<k m, m \leq n$. Daí, por escolha de $\delta_{\varepsilon}, d\left(\left(f^{k}\right)^{m-1}(x),\left(f^{k}\right)^{m-1}(y)\right)>\delta_{\varepsilon}$, com $m \leq n$. Logo, $S$ é $\left(n, \delta_{\varepsilon}\right)$-separado para $f^{k}$, o que implica:

$$
r\left(n, \delta_{\varepsilon}, f^{k}\right) \geq r(n k, \varepsilon, f)
$$

Das equações (1.1) e (1.2) temos :

$$
\frac{r(n k, \varepsilon, f)}{n} \leq \frac{r\left(n, \delta_{\varepsilon}, f^{k}\right)}{n} \leq \frac{r\left(n k, \delta_{\varepsilon}, f\right)}{n} .
$$

Fazendo $n \rightarrow \infty$,

$$
k h(\varepsilon, f) \leq h\left(\delta_{\varepsilon}, f^{k}\right) \leq k h\left(\delta_{\varepsilon}, f\right) .
$$

Como $\varepsilon \rightarrow 0$ faz com que $\delta_{\varepsilon} \rightarrow 0$, temos o que queríamos:

$$
h\left(f^{k}\right)=k h(f) .
$$

Quando caracterizamos um sistema dinâmico por um número, para que esta caracterização seja "boa"precisamos que este número seja um invariante por conjugações. O próximo resultado nos diz que entropia topológica é um invariante.

Teorema 1.1.23. Sejam $F: X \rightarrow X$ e $f: Y \rightarrow Y$ aplicações contínuas, onde $X$ e $Y$ são espaços métricos com métricas $d$ e $d^{\prime}$, respectivamente. Suponhamos que exista um homeomorfismo $k: X \rightarrow Y$ que seja uma conjugação topológica entre $F$ e $f$. Então, $h(f)=h(F)$.

Definição 1.1.24. Definimos $\Sigma_{N}$ como sendo o espaço das sequências com $N$ símbolos. Isto é,

$\Sigma_{N}=\left\{\left(\ldots x_{-2} x_{-1} x_{0} x_{1} x_{2} \ldots\right)\right.$, para $x_{i}$ pertencente a um conjunto de $N$ símbolos,$\left.i \in \mathbb{Z}\right\}$ Assim, definimos a aplicação shift, $\sigma: \Sigma_{N} \rightarrow \Sigma_{N}$, como sendo o deslocamento da sequência uma casa a esquerda:

$$
\sigma(x)=y \Leftrightarrow y_{i-1}=x_{i}, i \in \mathbb{Z}
$$


Sabemos que em muitos sistemas dinâmicos, podemos introduzir uma dinâmica simbólica, isto é, podemos exibir uma conjugação entre um sistema dinâmico e a aplicação shift, agindo sobre $\Sigma_{N}$. Visando isto, o próximo resultado explicita a entropia da aplicação shift.

Proposição 1.1.25. Seja $\sigma: \Sigma_{N} \rightarrow \Sigma_{N}$ a aplicação shift sobre o espaço das sequências com $N$ simbolos. Então,

$$
h(\sigma)=\log N
$$

Demonstração. Consideremos a seguinte métrica $d$ sobre $\Sigma_{N}$ :

$$
d(x, y)=2^{-n} \text {, onde } n \text { é o maior possível, tal que } x_{i}=y_{i} \text { para }|i|<n \text {. }
$$

Seja $\varepsilon=2^{-1}$. Assim, dois pontos $s, t \in \Sigma_{N}$ estão $\varepsilon-$ próximos se, e somente se, $s_{0}=t_{0}$. Da mesma forma, $\sigma^{j}(s)$ está $\varepsilon$-próximo de $\sigma^{j}(t)$ se, e somente se, $s_{j}=t_{j}$, para $0 \leq j<n$. Como existem $N^{n}$ escolhas possíveis de blocos $\left(s_{0}, \ldots, s_{n-1}\right)$, temos que

$$
r\left(n, 2^{-1}, \sigma\right)=N^{n}
$$

Daí,

$$
h\left(2^{-1}, \sigma\right)=\log N
$$

Agora, suponhamos $\varepsilon=2^{-k}$, para $k \geq 0$. Seguindo o mesmo raciocínio anterior, dado $s, t \in \Sigma_{N}, d(s, t) \leq 2^{-k}$ se, e somente se, $s_{j}=t_{j}$ para $|j|<k$. Então, $d\left(\sigma^{i}(s), \sigma^{i}(t)\right)>2^{-k}$ para algum $0 \leq i<n$ se, e somente se, $s_{j} \neq t_{j}$ para algum $0 \leq j<n+k$. Portanto,

$$
r\left(n, 2^{-k}, \sigma\right)=r\left(n+k, 2^{-1}, \sigma\right) .
$$

Daí,

$$
\frac{\log r\left(n, 2^{-k}, \sigma\right)}{n}=\frac{(n+k)}{n} \frac{\log r\left(n+k, 2^{-1}, \sigma\right)}{n+k},
$$

e fazendo $n \rightarrow \infty$ :

$$
h\left(2^{-k}, \sigma\right)=h\left(2^{-1}, \sigma\right)=\log N, \text { para todo } k \geq 0 .
$$

Portanto,

$$
h(\sigma)=\lim _{\varepsilon^{+} \rightarrow 0} h(\varepsilon, \sigma)=\log N .
$$

\subsection{Propriedades Ergódicas}

\subsubsection{Entropia Métrica}

Na seção anterior, a entropia topológica fornece uma maneira de medir quão um sistema é caótico, sobre um ponto de vista topológico. Nesta seção, vamos tentar dar uma definição de 
entropia que observe o sistema sob o ponto de vista de alguma medida invariante para este. Chamamos esta definição, de entropia métrica. No final da seção fazemos uma ligação entre estas duas definições.

Exemplos e propriedades sobre entropia métrica, podem ser encontradas em [9].

Definição 1.2.1. Seja $f: X \rightarrow X$ uma transformação que preserva a probabilidade $\mu$. Dada uma partição $\mathcal{Q}$ enumerável de $X$, a entropia da partição $\mathcal{Q}$ com respeito à $\mu$ é o número

$$
H_{\mu}(\mathcal{Q})=-\sum_{Q \in \mathcal{Q}} \mu(Q) \log \mu(Q)
$$

onde se convenciona que $0 \log 0=0$.

A partir de partições $\mathcal{P}$ e $\mathcal{Q}$ podemos definir uma nova partição

$$
\mathcal{P} \vee \mathcal{Q}=\{A \cap B ; A \in \mathcal{P} \text { e } B \in \mathcal{Q}\}
$$

Assim, dado uma partição $\mathcal{P}$, denotamos por $\mathcal{P}^{n}$ a partição

$$
\mathcal{P}^{n}=\bigvee_{i=0}^{n-1} f^{-i}(\mathcal{P})
$$

Definimos a entropia da partição $\mathcal{P}$ com respeito à transformação $f$ e à medida $\mu$ como sendo o número:

$$
h_{\mu}(f, \mathcal{P})=\lim _{n \rightarrow \infty} \frac{H_{\mu}\left(\mathcal{P}^{n}\right)}{n} .
$$

Definimos agora, uma forma de medir a complexidade de uma transformação, no sentido métrico.

Definição 1.2.2. A entropia de $f$ com respeito à medida $\mu$ é:

$$
h_{\mu}(f)=\sup _{\mathcal{P}} h_{\mu}(f, \mathcal{P})
$$

onde o supremo é tomado sobre todas as partições finitas de $X$.

Como prometido, o próximo resultado relaciona a entropia topológica com a entropia métrica.

Teorema 1.2.3 (Princípio Variacional). Seja $f: X \rightarrow X$ uma função contínua sobre o espaço métrico compacto $X$. Denote por $\mathcal{I}$, o conjunto das medidas (de probabilidades) invariantes por $f$. Então, vale a igualdade:

$$
h(f)=\sup _{\mu \in \mathcal{I}} h_{\mu}(f)
$$




\subsubsection{Estado de Equilíbrio e a função $\phi^{u}$}

Para falarmos de estado de equilíbrio precisamos, primeiro, definir o conceito de pressão topológica de uma função $f: X \rightarrow X$, com respeito a um observável $\phi: X \rightarrow \mathbb{R}$. Assim como para entropia, podemos definir o conceito de pressão topológica usando partições, e depois relacioná-lo com medidas invariantes, isto é, provar um análogo ao Princípio Variacional 1.2.3. No entanto, visando a não complicação do texto por notações, usaremos o Princípio Variacional, que pode ser encontrado em [6], para definirmos pressão topológica.

Não só a definição de pressão topológica através de partições, como todo o conteúdo desta seção, podem ser encontrados em [6].

Assim, definimos pressão topológica por

$$
P_{f}(\phi)=\sup _{\mu}\left(h_{\mu}(f)+\int \phi d \mu\right)
$$

onde $\mu$ percorre $M_{f}(X)$, isto é, o conjunto das medidas de probabilidades invariantes por $f$.

Observemos que o conceito de pressão topológica generaliza o conceito de entropia topológica. De fato, pelo Princípio Variacional 1.2.3, $h(f)=P_{f}(0)$, onde 0 é a função nula.

Definição 1.2.4. Seja $\mu \in M_{f}(X)$ satisfazendo $h_{\mu}(f)+\int \phi d \mu=P_{f}(\phi)$, então $\mu$ é chamado de estado de equilibrio para $\phi$.

Lembremos agora, que $\phi$ é Holder continua se existem constantes $a, \theta>0$ tal que :

$$
|\phi(x)-\phi(y)| \leq a d(x, y)^{\theta}
$$

Teorema 1.2.5. Seja $\Omega_{s}$ um conjunto básico hiperbólico para um difeomorfismo $f$, Axioma $A$, e $\phi: \Omega_{s} \rightarrow \mathbb{R}$ Holder continua. Então, $\phi$ possui um único estado de equilíbrio $\mu_{\phi}$. E, além disto, $\mu_{\phi}$ é ergódico.

Proposição 1.2.6. Seja $\Omega_{s}$ um conjunto básico hiperbólico para um difeomorfismo $f$, Axioma $A, e \phi, \psi: \Omega_{s} \rightarrow \mathbb{R}$ duas funções Holder contínuas. Então, os seguintes itens são equivalentes:

(a) $\mu_{\phi}=\mu_{\psi}$

(b) Existe constante $K$ tal que $S_{m} \phi(x)-S_{m} \psi(x)=k m$ quando $x \in \Omega_{s}$ e $f^{m}(x)=x$. Onde

$$
S_{m} \phi(x)=\sum_{j=0}^{m-1} \phi\left(f^{j}(x)\right) .
$$

Consideremos, agora, $M$ uma variedade com estrutura Riemanianna. Assim, esta induz uma medida de volume $m$ sobre $M$. Seja $f \in \operatorname{Diff}^{2}(M)$, Axioma A.

Definimos $\phi^{u}(x)=-\log \bar{\lambda}(x)$, onde $\bar{\lambda}(x)$ é o valor absoluto do Jacobiano, da aplicação $f$ restrita a direção instável $\mathbb{E}^{u}$. Isto é, $\bar{\lambda}(x)$ é o valor absoluto do determinante da aplicação linear

$$
D f: \mathbb{E}_{x}^{u} \rightarrow \mathbb{E}_{f(x)}^{u}
$$


Um conjunto básico hiperbólico $\Omega_{s}$ é um atrator se ele possui uma vizinhança captadora $U$ tal que $f(U) \subset U$, e

$$
\Omega_{s}=\bigcap_{n \geq 0} f^{n}(U) .
$$

Teorema 1.2.7. Seja $\Omega_{s}$ um conjunto básico hiperbólico $C^{2}$. Os seguintes itens são equivalentes:

(a) $\Omega_{s}$ é um atrator

(b) $m\left(W^{s}\left(\Omega_{s}\right)\right)>0$

(c) $P_{f \mid \Omega_{s}}\left(\phi^{u}\right)=0$. 



\begin{tabular}{|l|l|}
\hline Capítulo \\
\cline { 2 - 2 } & 2 \\
\hline
\end{tabular}

\section{Preliminares de Perturbação}

Chamamos o par $(M, \omega)$ de variedade simplética, se $M$ possui dimensão $2 n$ e $\omega$ é uma 2forma diferenciável, $C^{\infty}$, fechada, e não degenerada, sobre $M$. Onde não degenerada significa que se $x \in M$ e $v \in T_{x} M$ satisfaz $\omega(x)(v, w)=0$, para todo $w \in T_{x} M$, então $v=0$. Um difeomorfismo $f: M \rightarrow M$, é chamado de difeomorfismo simplético se $f$ preserva $\omega$. Isto é, $f^{*} \omega=\omega$, onde $f^{*} \omega$ é o pull-back de $\omega$. Sabendo que $f^{*} \omega=\operatorname{Jac} f \omega$, com Jac $f$ denotando o determinante do Jacobiano de $f$, temos que $f$ preserva $\omega$ se, e somente se, $\operatorname{Jac} f(x)=1$ para todo ponto $x \in M$. Definimos por $\operatorname{Diff}_{\omega}^{1}(M)$ o conjunto dos difeomorfismos simpléticos. Usaremos sobre este espaço a topologia uniforme $C^{1}$.

Neste capítulo mostramos uma maneira de realizar perturbações de difeomorfismos no mundo simplético. Apesar da generalidade dos resultados apresentados neste capítulo, como precisamos destes, apenas para $M$ de dimensão 2, e pensando em não complicar a notação, assumimos, sempre, que estamos trabalhando em dimensão 2. Note que neste caso a 2-forma $\omega$ é um elemento de área, e o tratamos assim muitas vezes. Também, como estamos interessados, apenas, em perturbações locais, podemos trabalhar, sem perda de generalidade, em $\mathbb{R}^{2}$. Antes de enunciarmos o lema de perturbação, definimos a principal ferramenta usada na perturbação de difeomorfismos simpléticos, a função geradora.

\subsection{Função Geradora}

Para construirmos a função geradora, precisamos de alguns resultados sobre formas diferenciais. 
Definição 2.1.1. Seja $U \subset \mathbb{R}^{n}$ um aberto. Uma 1-forma diferencial $\omega: U \rightarrow\left(\mathbb{R}^{n}\right)^{*}$ diz-se exata em $U$ caso exista uma função $f: U \rightarrow \mathbb{R}$ diferenciavel tal que $\omega=d f$. Isto é, se

$$
\omega=\sum_{i=1}^{n} a_{i} d x_{i} \Rightarrow a_{i}=\frac{\partial f}{\partial x_{i}}, \text { para todo } i=1, \ldots, n \text {. }
$$

A função $f$ tal que $\omega=d f$ chama-se a primitiva da 1-forma $\omega$. Se $U$ é conexo, duas primitivas da mesma forma $\omega$ diferem por uma constante.

Note que uma 1-forma $\omega=\sum_{i=1}^{n} a_{i} d x_{i}$ é exata se e somente se o campo de vetores $F=\left(a_{1}, \ldots, a_{n}\right)$ é o gradiente de uma função diferenciavel $f: U \rightarrow \mathbb{R}$. Neste caso, a função $f$ chama-se o potencial do campo $F$.

Definição 2.1.2. Uma 1-forma diferencial $\omega=\sum_{i=1}^{n} a_{i} d x_{i}$ é fechada se $d \omega=0$ ou equivalentemente quando $\frac{\partial a_{i}}{\partial x_{j}}=\frac{\partial a_{j}}{\partial x_{i}}$ para $i, j=1, \ldots, n$.

O conceito de forma exata pode ser conciderado localmente. Isto é, dada uma 1-forma $\omega$ dizemos que ela é localmente exata se para todo ponto $p$ em $U$ existe um aberto $V$, contendo $p$, tal que $\omega$ é exata em $V$.

Proposição 2.1.3. Uma 1-forma $\omega: U \rightarrow\left(\mathbb{R}^{n}\right)^{*}$ de classe $C^{1}$, é fechada se e somente se é localmente exata.

Demonstração. A demonstração é feita no caso $n=3$. O caso geral é inteiramente análogo.

Inicialmente mostramos que se $\omega$ é uma forma diferencial fechada numa bola $B$ então $\omega$ é exata em $B$. Para simplificarmos a notação supomos que $\omega=a d x+b d y+c d z$ é fechada na bola $B$, de centro na origem em $\mathbb{R}^{3}$. O caso geral segue analogamente. Como $\omega$ é fechada temos:

$$
a_{y}=b_{x}, \quad a_{z}=c_{x}, \quad b_{z}=c_{y}, \quad \text { onde } k_{j}=\frac{\partial k}{\partial j} \text { para } k=a, b, c \text { e } j=x, y, z
$$

Usando o fato de $B$ ser convexo o caminho $\lambda:[0,1] \rightarrow B, \lambda(t)=(t x, t y, t z)$, está bem definido para todo ponto $(x, y, z) \in B$. Assim, podemos definir $f: B \rightarrow \mathbb{R}$, pondo para cada $(x, y, z) \in B$

$$
f(x, y, z)=\int_{0}^{1}[a(t x, t y, t z) x+b(t x, t y, t z) y+c(t x, t y, t z) z] d t=\int_{\lambda} \omega
$$


Como $\omega$ é de classe $C^{1}$ as funções $a, b$ e $c$ são funções de classe $C^{1}$, logo podemos usar a Regra de Leibniz:

$$
\begin{aligned}
\frac{\partial f}{\partial x}(x, y, z) & =\int_{0}^{1}\left[a_{x}(t x, t y, t z) t x+a(t x, t y, t z)+b_{x}(t x, t y, t z) t y+c_{x}(t x, t y, t z) t z\right] d t \\
& =\int_{0}^{1}\left[\left(a_{x}(t x, t y, t z) x+a_{y}(t x, t y, t z) y+a_{z}(t x, t y, t z) z\right) t+a(t x, t y, t z)\right] d t \\
& =\int_{0}^{1}\left[\left((a \circ \lambda)^{\prime}(x, y, z)\right) t+a \circ \lambda(x, y, z)\right] d t \\
& =\int_{0}^{1}[((a \circ \lambda)(x, y, z)) t]^{\prime} d t \\
& =a(x, y, z) .
\end{aligned}
$$

Notemos que na segunda igualdade usamos o fato de $\omega$ ser fechada.

De modo análogo conseguimos

$$
\frac{\partial f}{\partial y}(x, y, z)=b(x, y, z) \text { e } \frac{\partial f}{\partial z}(x, y, z)=c(x, y, z)
$$

Portanto, temos que $d f=\omega$.

Reciprocamente, sabemos que $d d \alpha=0$ para toda forma diferencial $\alpha$ de classe $C^{2}$. Como $\omega$ é uma forma de classe $C^{1}$ localmente exata, dado uma bola $B \in U$ existe $f$ primitiva de $\omega$ em $B$, isto é, $d f=\omega$. Assim, temos que $f$ é de classe $C^{2}, \operatorname{logo} d d f=0$ e portanto $d \omega=0 \mathrm{em}$ $B$. Como isto pode ser feito localmente em $U$, temos que $d \omega=0$. Logo, a 1-forma diferencial $\omega$ é fechada.

Observação 2.1.4. Na Proposição 2.1 .3 foi necessário apenas a convexidade de $B$. Na verdade foi usado menos ainda, precisavamos apenas que $B$ fosse estrelado. Assim, toda 1-forma $\omega$ fechada num domínio aberto convexo no espaço $\mathbb{R}^{n}$ é uma forma exata.

\section{Construção da função geradora}

Suponhamos $(x, y)$ coordenadas em $\mathbb{R}^{2}$ e $f(x, y)=(\xi(x, y), \eta(x, y))$ um difeomorfismo de classe $C^{1}$ que preserva um elemento de área $\omega, \operatorname{com} f(0,0)=(0,0)$ e $\frac{\partial \eta}{\partial y} \neq 0$ em todo ponto.

Afirmação 1: Podemos encontrar y em função de $x$ e $\eta$ de maneira $C^{1}$ apartir da equação $\eta=\eta(x, y)$.

Como $f$ é um difeomorfismo temos que a aplicação $\eta: \mathbb{R}^{2} \rightarrow \mathbb{R}$ é sobrejetiva. Tomemos $\left(x_{0}, y_{0}\right) \in \mathbb{R}^{2}$ e seja $\eta_{0}=\eta\left(x_{0}, y_{0}\right)$. Como $\frac{\partial \eta}{\partial y}\left(x_{0}, y_{0}\right) \neq 0$, o Teorema da Função Implicita nos dá que existem vizinhanças $I$ de $x_{0}$ e $J$ de $y_{0}$ tal que $(\eta)^{-1}\left(\eta_{0}\right) \cap(I \times J)$ é gráfico de uma função $y\left(., \eta_{0}\right): I \rightarrow J$ de classe $C^{1}$, e

$$
y_{x}=\frac{\partial y}{\partial x}=\frac{-\frac{\partial \eta}{\partial x}}{\frac{\partial \eta}{\partial y}}=\frac{-\eta_{x}}{\eta_{y}}
$$


Fixando $x$ em $I$, temos que $\eta(x,):. J \rightarrow \mathbb{R}$ é uma aplicação $C^{1}$, e como $\frac{\partial \eta}{\partial y} \neq 0$,

$$
\eta(x, y) \neq \eta\left(x, y^{\prime}\right) \text { para todo } y, y^{\prime} \in J .
$$

De fato, se existisse $y<y^{\prime}$ tal que $\eta(x, y)=\eta\left(x, y^{\prime}\right)$ poderíamos usar o Teorema do Valor Médio e encontrar $y_{1} \in\left(y, y^{\prime}\right)$ tal que $\eta_{y}=0$. O que geraria uma contradição.

Assim, existe vizinhança $V$ de $\eta_{0}$ tal que

$$
\eta(x, .): J \rightarrow V \text { é um difeomorfismo } C^{1}, \operatorname{com} \eta_{y}=\frac{\partial \eta}{\partial y}(x, y) \neq 0 \text { todo } y \in J .
$$

Logo, a aplicação $y(x,):. V \rightarrow J$ é $C^{1}$, e $y_{\eta}=\frac{1}{\eta_{y}}$.

Com isto, a aplicação $y(x, \eta)$ é $C^{1}$ numa vizinhança de $\left(x_{0}, \eta_{0}\right)$, e como este ponto foi tomado arbitrariamente, concluímos a afirmação 1 .

Usando a Afirmação 1 , a aplicação $(x, \eta) \longmapsto(x, y(x, \eta))$ permite-nos usarmos $x$ e $\eta$ como coordenadas sobre $\mathbb{R}^{2}$.

Afirmação 2: A 1-forma diferencial $\alpha=\xi d \eta+y d x$ é fechada.

$$
\begin{aligned}
d \alpha & =\left(\xi_{x} d x+\xi_{y} y_{x} d x\right) d \eta+\left(y_{\eta}\right) d \eta d x \\
& =\left(\xi_{x}+\xi_{y} y_{x}-y_{\eta}\right) d x d \eta \\
& =\left(\xi_{x}-\xi_{y} \frac{\eta_{x}}{\eta_{y}}-\frac{1}{\eta_{y}}\right) d x d \eta \\
& =\left(\frac{\xi_{x} \eta_{y}-\xi_{y} \eta_{x}-1}{\eta_{y}}\right) d x d \eta \\
& =\left(\frac{\mathrm{Jac} f-1}{\eta_{y}}\right) d x d \eta,
\end{aligned}
$$

Na terceira igualdade foram usados resultados obtidos na demonstração da Afirmação 1. Como $f$ é um difeomorfismo que preserva área temos que Jac $f=1$ em todo ponto, logo $d \alpha=0$.

Como $\omega$ está definida em todo $\mathbb{R}^{2}$ e é de classe $C^{1}$, pela Proposição 2.1.3 temos que $\omega$ é exata, isto é, existe uma única $S: \mathbb{R}^{2} \rightarrow \mathbb{R}$, de classe $C^{2}$, tal que $d S=\omega$ e $S(0,0)=0$. Assim,

$$
S_{x}=y, \quad S_{\eta}=\xi \text { e } S_{x \eta}=\frac{\partial y}{\partial \eta} \neq 0 .
$$

Tal função $S$ é chamada de função geradora de $f$, e a denotamos por $S_{f}$.

Reciprocamente, se tomarmos $S(x, \eta)$ uma função de classe $C^{2}$ tal que $S(0,0)=0$ e $S_{x \eta} \neq 0$, usando o mesmo argumento da Afirmação 1, podemos encontrar $\eta$ em função de $x$ e $y$ na equação $S_{x}(x, \eta)=y$. Assim, tomando a 1-forma $\omega=S_{\eta} d \eta+y d x$ temos que $\omega$ é fechada, e por um processo similar ao da Afirmação 2, podemos mostrar que $S_{\eta x} \eta_{y}-S_{\eta \eta} \eta_{x}=1$ em todo ponto. Assim,

$$
f(x, y)=\left(S_{\eta}(x, \eta(x, y)), \eta(x, y)\right)
$$


tem Jacobiano 1 em todo ponto. Logo preserva área.

Notemos que o conceito de função geradora é local. Assim, quando falarmos em proximidade de funções geradoras estamos falando, sempre, localmente. Usando o teorema seguinte, que pode ser encontrado em [11], podemos estender este conceito, e resultados, para variedades simpléticas.

Teorema 2.1.5. Seja $(M, \omega)$ uma variedade simplética. Para todo ponto $x \in M$ existe vizinhança $U$ de $x$ e coordenadas $\phi: U \rightarrow \mathbb{R}^{2 n}$ tal que para todo ponto $y \in U$, w está na forma estandarte com respeito a base $\left\{\frac{\partial}{\partial x_{1}}, \ldots, \frac{\partial}{\partial x_{2 n}}\right\}$. Isto é,

$$
\phi^{*}\left(\sum_{i=1}^{n} d x_{i} \wedge d x_{n+i}\right)=\omega .
$$

Estas coordenadas são chamadas de coordenadas de Darboux ou simpléticas.

Exemplo 2.1.6. Consideremos a aplicação $g: \mathbb{R}^{2} \rightarrow \mathbb{R}^{2}, g(x, y)=(x, y+\varepsilon x)$, com $\varepsilon \in \mathbb{R}$. No caso, temos $\xi(x, y)=x$ e $\eta(x, y)=y+\varepsilon x$. Assim,

$$
D g(x, y)=\left[\begin{array}{ll}
1 & 0 \\
\varepsilon & 1
\end{array}\right],
$$

e, portanto, é um difeomorfismo que preserva área, com $\eta_{y}(x, y) \neq 0$, para todo $(x, y)$. Neste caso faz sentido em falar de $S_{g}$, a função geradora para $g$.

Como $S=S_{g}$ é geradora, ela tem que satisfazer: $S_{x}(x, \eta)=y(x, \eta)$ e $S_{\eta}(x, \eta)=$ $\xi(x, y(x, \eta))$, onde $y(x, \eta)=\eta-\varepsilon x$. Integrando a primeira destas temos:

$$
S(x, \eta)=\eta x-\frac{\varepsilon x^{2}}{2}+K(\eta)
$$

onde $K(\eta)$ é uma função que depende somente de $\eta$. E assim, derivando esta em relação a $\eta$,

$$
S_{\eta}(x, \eta)=x+K^{\prime}(\eta)
$$

Como, $S_{\eta}(x, \eta)=\xi(x, y(x, \eta))=x$ temos que $K(\eta)=K$, para algum $K$ constante. Impondo que $S(0,0)=0$, temos que $K=0$. E, portanto, concluímos que a função geradora $S_{g}$, tal que $S_{g}(0,0)=0$ é

$$
S_{g}(x, \eta)=x \eta-\frac{\varepsilon x^{2}}{2}
$$

Notemos que para $\varepsilon=0$, a função $g$ é a identidade $I d$. E assim, a geradora da identidade $I d$, é $S_{i d}(x, \eta)=x \eta$. 


\subsection{Lema de Perturbação}

O interessante de se trabalhar em variedades simpléticas é que funções simpléticas são $C^{1}$ próximas se, e somente se, suas geradoras são $C^{2}$ próximas. Sendo assim, basta realizarmos boas perturbações sobre as geradoras para conseguirmos perturbações desejadas de difeomorfismos simpléticos.

Lema 2.2.1. Um difeomorfismo $f \in \operatorname{Diff}_{\omega}^{1}(M)$ é $C^{1}$ próximo da aplicação identidade Id se, e somente se, $S_{f}$ é $C^{2}$ próximo de $S_{I d}$.

Demonstração. Suponhamos $f(x, y)=(\xi(x, y), \eta(x, y))$. Usando a Proposição 2.1.3, podemos explicitar a função geradora $S_{f}$ da seguinte forma:

$$
S_{f}(x, \eta)=\int_{0}^{1}[\xi(t x, t y(x, \eta)) \eta+y(t x, t \eta) x] d t .
$$

Agora, supondo $f C^{1}$ próxima da $I d$, temos que $\eta(x, y) \approx^{C^{1}} y$, daí $y(x, \eta) \approx^{C^{1}} \eta$. Além disto, $\xi(x, y) \approx C^{1} x$. Usando isto,

$$
\xi(t x, t y(x, \eta)) \eta+y(t x, t \eta) x \approx^{C^{1}} 2 t x \eta, \text { para todo } 0 \leq t \leq 1
$$

E assim,

$$
S_{f}(x, \eta) \approx^{C^{2}} \int_{0}^{1} 2 t x \eta d t=x \eta .
$$

Pelo Exemplo 2.1.6, sabemos que $S_{i d}(x, \eta)=x \eta$. Logo, temos que $S_{f}$ e $S_{i d}$ são realmente $C^{2}$ próximas.

Reciprocamente, se $S_{f}(x, \eta)$ e $x \eta$ são $C^{2}$ próximas então,

$$
f(x, y)=\left(\left(S_{f}\right)_{\eta}(x, \eta(x, y)), \eta(x, y)\right) \approx^{C^{1}}(x, y) .
$$

Ou seja, $f$ e $I d$ são $C^{1}$ próximas.

E assim, usando o Lema 2.2.1, podemos demonstrar o seguinte Lema de Perturbação:

Lema 2.2.2. Dado $\epsilon>0$, existe constante $K>0$ tal que os seguintes fatos acontecem:

a) Se $g: \mathbb{R}^{2} \rightarrow \mathbb{R}^{2}$, de classe $C^{1}$, preserva área e $g(0,0)=(0,0)$, é $K^{-1} \varepsilon-C^{1}$ próximo da identidade Id em $B(0, r)$, então existe $h: \mathbb{R}^{2} \rightarrow \mathbb{R}^{2}, \varepsilon-C^{1}$ próxima da Id, tal que $h \mid B\left(0, \frac{r}{2}\right)=g$ e $h \mid(B(0, r))^{c}=I d$. Onde $B(0, r)$ é a bola de centro na origem e raio $r$.

b) Dado $A: \mathbb{R}^{2} \rightarrow \mathbb{R}^{2}$ uma aplicação linear simplética, tal que $\|A-I d\|<K^{-1} \varepsilon$, existe $h: \mathbb{R}^{2} \rightarrow \mathbb{R}^{2}, \varepsilon-C^{1}$ próxima da Id, satisfazendo $h(0)=0$, Dh $(0)=A$ e $h=I d$ fora de uma vizinhança da origem.

Demonstração. Parte (a)-Seja $g(x, y)=(\xi(x, y), \eta(x, y))$, como na hipótese. 
Consideremos agora $\beta: \mathbb{R} \rightarrow[0,1]$ uma função bump, isto é, uma função real $C^{\infty}$, tal que:

$$
\beta(x)= \begin{cases}1, & \text { para } x \leq \frac{1}{2} \\ 0, & \text { para } x \geq 1\end{cases}
$$

Assim, tomemos

$$
K^{\prime}=\sup _{x \in \mathbb{R}}\left\{1,|\beta(x)|, \mid \beta^{\prime \prime}(x)\right\} .
$$

Para não complicarmos a notação tratamos como sendo iguais as bolas com centro na origem, estando a olhar para $\mathbb{R}^{2}$ tanto nas coordenadas $(x, y)$ quanto nas coordenadas $(x, \eta)$.

Tomemos,

$$
S(x, \eta)=\beta\left(\frac{|(x, \eta)|}{r}\right)\left(S_{g}(x, \eta)-x \eta\right)+x \eta .
$$

Como $\beta$ é $C^{\infty}$, temos que $S$ possui a mesma classe de diferenciabilidade que $S_{g}(x, \eta)$ e $x \eta$, ou seja, $S$ é $C^{2}$. Mais ainda, usando a hipótese, o Lema 2.2.1 e a escolha de $K^{\prime}$ temos que:

$$
\|S(x, \eta)-x \eta\|_{2} \leq K^{\prime} c \frac{\varepsilon}{K}
$$

onde $c$ é uma constante que depende apenas de $r$, e $\|\cdot\|_{2}$ é a norma na topologia $C^{2}$. Assim, usando novamente o Lema 2.2.1, temos que o difeomorfismo simplético $h$, gerado por $S$, é $K^{\prime} c K^{-1} \varepsilon-C^{1}$ próximo da aplicação $I d$. Além disto, por construção de $S, h \mid B\left(0, \frac{r}{2}\right)=g$ e $h \mid(B(0, r))^{c}=I d$.

Portanto, tomando $K=K^{\prime} c$ temos o que queríamos.

Parte (b) Tomando $g(x)=A x$, a parte (b) sai direto da parte $(a)$. 



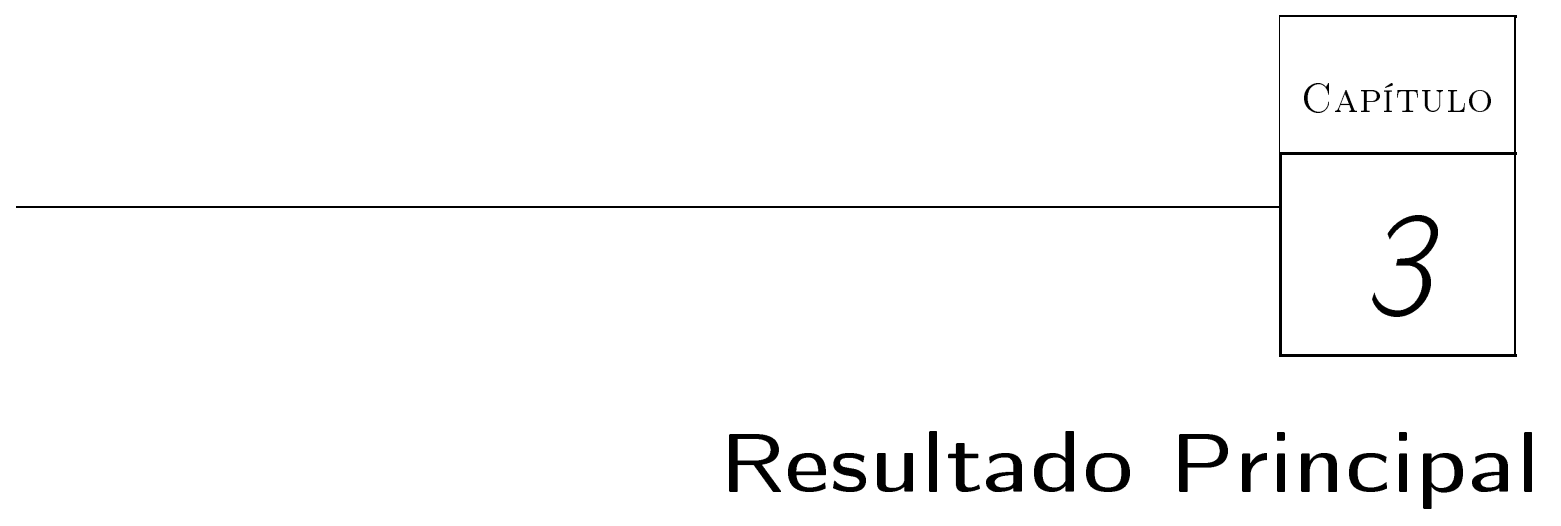

Neste capítulo, consideramos $(M, \omega)$ uma variedade simplética, com $M$ de dimensão 2, e provamos duas propriedades genéricas para $\operatorname{Diff}_{\omega}^{1}(M)$, sobre entropia e dimensão de Hausdorff. Como podemos ver no Apêndice, $\operatorname{Diff}_{\omega}^{1}(M)$ é um espaço de Baire, logo conjuntos genéricos ainda são densos.

Antes de enunciarmos o Resultado Principal, definimos Dimensão de Hausdorff e o funcional $s($.$) sobre Diff { }_{\omega}^{1}(M)$, o qual vai nos dar uma limitação inferior para entropia . Ressaltamos que estamos olhando para $\operatorname{Diff}_{\omega}^{1}(M)$ com a topologia uniforme $C^{1}$.

\subsection{Dimensão de Hausdorff e o funcional $s($.}

Seja $E$ um subconjunto fechado de $M$ e $\alpha, \epsilon>0$ números reais positivos. Agora, seja $H_{\epsilon}^{\alpha}(E)=\inf \left\{\sum_{i}\left(\operatorname{diam} U_{i}\right)^{\alpha}:\left\{U_{i}\right\}\right.$ é uma cobertura aberta de $E$ onde cada elemento possui diâmetro menor do que $\epsilon\}$.

A medida $\alpha$-dimensional de Hausdorff de $E$ é definida como sendo o número

$$
H^{\alpha}(E)=\lim _{\epsilon \rightarrow 0} H_{\epsilon}^{\alpha}(E)
$$

Como $H_{\varepsilon_{1}}^{\alpha}(E) \leq H_{\varepsilon_{2}}^{\alpha}(E)$ para $\varepsilon_{1}>\varepsilon_{2}$, o limite acima existe, mas pode ser igual a infinito. Se $A \subset \mathbb{R}^{n}$, então $H^{n}(A)$ é a medida de Lebesgue de $A$. 
A dimensão de Hausdorff de $E$, denotada por $H D(E)$, é definida como sendo o número $d$ se

$$
H^{p}(A)= \begin{cases}\infty & \text { para } 0 \leq p<d \\ 0 & \text { para } d<p \leq \infty\end{cases}
$$

Observação 3.1.1. Para um conjunto $A \subset \mathbb{R}^{n}$, temos que

$$
H D(A) \geq \operatorname{dim} A,
$$

onde $\operatorname{dim} A$, é a dimensão topológica.

Vamos agora definir o funcional $s($.$) .$

Lembramos que $\tau(p)$ denota o período do ponto periódico $p$, para $f$. Se $f \in \operatorname{Diff}_{\omega}^{1}(M)$, como a dimensão de $M$ é dois, e o Jac $f(x)=1$ para todo $x \in M$, dado um ponto periódico hiperbólico $p, D f_{p}^{\tau(p)}$ possui um único autovalor de norma maior do que um, o qual denotamos por $\lambda(p)$. Notemos ainda, que o outro autovalor é $1 / \lambda(p)$.

Seja $f \in \operatorname{Diff}_{\omega}^{1}(M)$. Dado um inteiro $n>0$ positivo, consideremos $\operatorname{Hip}_{n} f$ como sendo o conjunto dos pontos periódicos hiperbólicos de $f$ com período menor ou igual a $n$.

Assim, denotemos:

$$
s_{n}(f)=\max \left\{\frac{1}{\tau(p)} \log |\lambda(p)|, \quad p \in \operatorname{Hip}_{n} f\right\} .
$$

Como $\operatorname{Hip}_{n} f \subset \operatorname{Hip}_{n+1} f$, temos que $s_{n}(f) \leq s_{n+1}(f)$.

Sendo assim, pela monotonicidade de $s_{n}$, definimos:

$$
s(f)=\lim _{n \rightarrow \infty} s_{n}(f) .
$$

Observação 3.1.2. Observemos que o número real $s_{n}(f)$, é nada mais nada menos, que o maior dos expoentes de Liapunov sobre os pontos periódicos hiperbólicos de período menor ou igual a $n$, restrito aos vetores do subfibrado instável.

Lema 3.1.3. O funcional $s_{n}($.$) é contínuo, para n \geq 0$.

Demonstração. Seja $f \in \operatorname{Diff}_{\omega}^{1}(M)$. Notemos que é possível $\sharp H y p_{n} f$ ser infinito. No entanto, pelo o Teorema 1.1.12, temos que tais pontos periódicos devem se acumular em pontos periódicos não hiperbólicos. Como $\operatorname{Jac}(f(x))=1$ em todo ponto, temos que tais pontos periódicos possuem autovalores com valores absolutos iguais a um. Sendo assim, apenas um número finito de pontos periódicos hiperbólicos são relevantes no cálculo de $s_{n}(f)$. Tomando $g$ numa vizinhança pequena de $f$ em $\operatorname{Diff}_{\omega}^{1}(M)$, temos que os pontos periódicos hiperbólicos em $\operatorname{Hyp}_{n}(g)$ que importam no cálculo de $s_{n}(g)$ são os pontos periódicos que são continuação daqueles que importavam no caso $f$. Agora, como estes são continuação dos anteriores temos que

$$
\left|s_{n}(g)-s_{n}(f)\right| \leq \varepsilon, \text { para } g \in U,
$$

onde $U$ é uma vizinhança pequena de $f$ em $\operatorname{Diff}_{\omega}^{1}(M)$. 


\subsection{Propriedades genéricas para $\operatorname{Diff}_{\omega}^{1}(M)$}

Ressaltamos que nesta seção, a menos que seja especificado o contrário, $(M, \omega)$ ainda é uma variedade simplética, com $M$ sendo uma variedade, $C^{\infty}$, compacta, conexa, de dimensão 2 .

Munido de todas as definições necessárias, podemos enunciar o resultado principal deste trabalho.

Teorema 3.2.1. Existe um conjunto residual $\mathcal{B} \subset \operatorname{Diff}_{\omega}^{1}(M)$ tal que se $f$ está em $\mathcal{B}$, então cada conjunto $\overline{H(p, f)}$ tem dimensão de Hausdorff dois. Mais ainda, se f está em $\mathcal{B}$ e não é Anosov, então

$$
h(f) \geq s(f) .
$$

Observação 3.2.2. Seja $f \in \operatorname{Diff}_{\omega}^{1}(M)$, um difeomorfismo de Anosov. Seja $x \in M$, um ponto qualquer. Toda vizinhança de $x$ possui medida positiva, com respeito a $\omega$. Assim, usando o Teorema de Recorrência de Poincaré, [9], para cada vizinhança $U$ de $x$, existe $q \in U$ e inteiro positivo $n$, tal que $f^{n}(q) \in U$. Desta forma, $x$ está no conjunto dos pontos não errantes $\Omega(f)$. Logo, $M=\Omega(f)$. Com isto, mais o fato de $f$ ser Anosov, podemos ver em [4], que toda variedade estável é densa em $M$. Usando os mesmos resultados para $f^{-1}$, temos que toda variedade instável, também é densa em $M$. Como $M$ é hiperbólico, as interseções destas também são densas e transversais. Em particular, $\overline{H(p, f)}=M$, para todo ponto $p$ periódico hiperbólico. E assim, a primeira parte do Teorema 3.2.1 é trivialmente satisfeita.

Para demonstrarmos o Teorema 3.2.1, precisamos de uma série de resultados.

Comecemos citando alguns resultados conhecidos, contendo propriedades genéricas para difeomorfismos simpléticos.

Os dois próximos resultados são devidos a Newhouse, [2].

Teorema 3.2.3. Para $M$ uma variedade compacta simplética, existe um subconjunto residual $\mathcal{B} \subset \operatorname{Diff}_{\omega}^{1}(M)$ tal que se $f \in \mathcal{B}$, então $f$ é Anosov ou os pontos periódicos 1-elípticos de $f$ são densos em $M$.

Para efeito de curiosidade, fugindo um pouco do nosso contexto, sabemos que a esfera $S^{2}$ não admite difeomorfismos de Anosov. Assim, pelo Teorema 3.2.3, existe um subconjunto residual em Diff ${ }_{\omega}^{1}\left(S^{2}\right)$ tal que para todo difeomorfismo $f$ neste conjunto, os pontos periódicos 1-elípticos de $f$ são densos em $S^{2}$.

Proposição 3.2.4. Se $M$ é uma variedade compacta simplética, existe um subconjunto residual $\mathcal{B} \subset \operatorname{Diff}_{\omega}^{1}(M)$ tal que para $f \in \mathcal{B}$, os pontos periódicos hiperbólicos de $f$ são densos em $M$.

O Teorema seguinte, devido a Takens [3], é muito útil na construção de uma tangência homoclínica para um ponto periódico hiperbólico, de um difeomorfismo não anosov. 
Teorema 3.2.5. Seja $M$ uma variedade compacta de dimensão 2, com um elemento de área $\omega$. Então existe um subconjunto residual $\mathcal{B} \subset \operatorname{Diff}_{\omega}^{1}(M)$ tal que se $f \in \mathcal{B}$, e p é um ponto periódico hiperbólico para $f$, então $W^{u}(p, f) \cap W^{s}(p, f)$ é denso tanto em $W^{u}(p)$, quanto em $W^{s}(p)$.

A próxima proposição é a peça chave na demonstração do Teorema 3.2.1.

Seja $\mathcal{A} \subset \operatorname{Diff}_{\omega}^{1}(M)$, o conjunto dos difeomorfismos de Anosov sobre $M$, e seja $\mathcal{D}=$ $\operatorname{Diff}_{\omega}^{1}(M)-\mathcal{A}$.

Proposição 3.2.6. Seja $p$ um ponto periódico hiperbólico para um difeomorfismo $f \in \mathcal{D}$, tal que $W^{u}(o(p))$ é tangente a $W^{s}(o(p))$ em algum ponto. Dado $\varepsilon>0$, para toda vizinhança $\mathcal{N}$ de $f$ em $D$, existe $g \in \mathcal{N}$ tal que $p$ é um ponto periódico hiperbólico para $g$, e

a) g tem um conjunto básico hiperbólico $\Lambda$ em $\overline{H(p, g)}$ para o qual

$$
h(g \mid \Lambda)>\frac{1}{\tau(p)} \log |\lambda(p)|-\varepsilon
$$

b) cada $g_{i}$ próximo de $g$ tem um conjunto básico hiperbólico $\Lambda\left(g_{i}\right)$ em $\overline{H\left(p\left(g_{i}\right), g_{i}\right)}$, onde $p\left(g_{i}\right)$ é a continuação do ponto $p$, tal que $H D\left(\Lambda\left(g_{i}\right)\right)>2-\varepsilon$.

Demonstração. Parte (a)

Assumimos, primeiro, para simplificar a notação que $p$ é um ponto fixo de $f$, isto é, $\tau(p)=1$.

Para um conjunto $E$ e um ponto $z \in E$, definimos $C(z, E)$ a componente conexa de $E$ que contém $z$.

Suponhamos que $W^{u}(p, f)$ tangencia $W^{s}(p, f)$ em $z_{0}$. Introduzindo coordenadas simpléticas, Teorema 2.1.5, existe vizinhança $U$ de $z_{0}$, e coordenadas $\phi: U \rightarrow \mathbb{R}^{2}$, tal que $\phi^{*}(d x \wedge d y)=\omega, \phi\left(z_{0}\right)=(0,0)$ e $\phi\left(C\left(z_{0}, W^{s}(p, f) \cap U\right)\right)$ está contida no eixo $x$. Figura 3.1. Tomemos $U$ como sendo um domínio fundamental. Isto é, $f^{-1}(U) \cap U=\emptyset$.

Pelo Teorema da Variedade Estável 1.1.14, diminuindo $U$ se necessário, $\phi\left(C\left(z_{0}, W^{u}(p, f) \cap\right.\right.$ $U)$ ) é gráfico de uma função de classe $C^{1}, g: \mathbb{R} \rightarrow \mathbb{R}$, isto é,

$$
\phi\left(C\left(z_{0}, W^{u}(p, f) \cap U\right)\right)=\left\{(x, g(x)) ; x \in \phi\left(C\left(z_{0}, W^{s}(p, f) \cap U\right)\right)\right\} .
$$

Tomando $r(x, y)=(x, y-g(x))$, como

$$
\operatorname{Jac} r(x, y)=\operatorname{det}\left[\begin{array}{cc}
1 & 0 \\
-g^{\prime}(x) & 1
\end{array}\right]=1,
$$

para todo $(x, y), r$ é um difeomorfismo simplético da mesma classe de diferenciabilidade que $g$, logo de classe $C^{1}$.

Dado $\gamma>0$ qualquer, como $W^{s}(p, f)$ e $W^{u}(p, f)$ são tangentes em $z_{0}$, podemos reduzir $U$, se necessário, tal que $g(x)$ seja $\gamma-C^{1}$ próximo da função nula, $y \equiv 0$, em $\phi(U)$. Desta forma, temos que $r$ é $\gamma-C^{1}$ próximo da função identidade $I d$. 


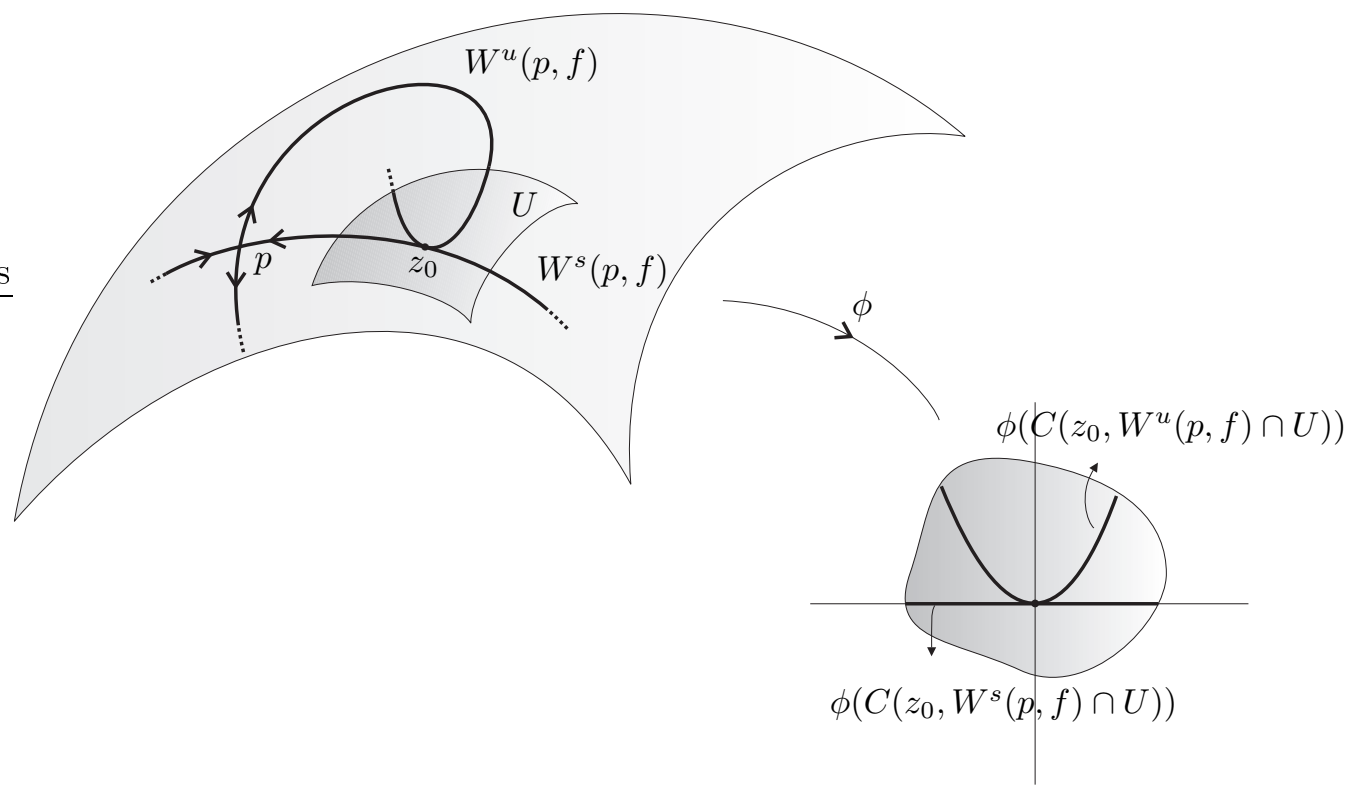

Figura 3.1:

Assim, dado $\varepsilon>0$ e usando o item (a) do Lema de Perturbação 2.2.2, existe um difeomorfismo simplético $h, \varepsilon-C^{1}$ próximo da $I d$, e $h=r$ numa vizinhança da origem.

Notemos que $r$ faz com que $\phi\left(C\left(z_{0}, W^{s}(p, f) \cap U\right)\right.$ coincida num intervalo $J \ni(0,0)$ com $\phi\left(z_{0}, W^{u}(p, f) \cap U\right)$. Figura 3.2.

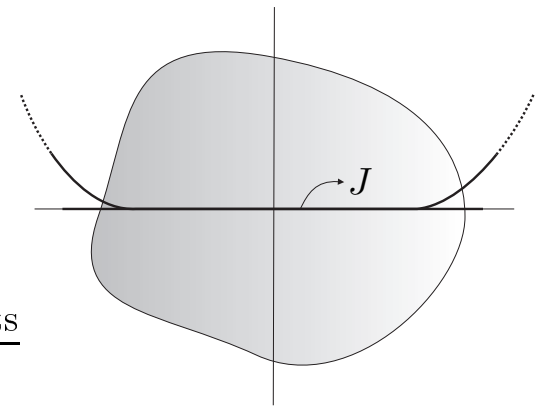

Figura 3.2:

Como $h$ é uma perturbação local, podemos olhar para $h$ como sendo uma perturbação da $I d$ em $U$, no espaço $\operatorname{Diff}_{\omega}^{1}(M)$. Olhemos, agora, para a função $h \circ f$. Por ser uma composição de difeomorfismos simpléticos, ainda é um difeomorfismo simplético; e além disso, é $\epsilon-C^{1}$ próximo de $f$, sendo idêntico a $f$ em $\left(f^{-1}(U)\right)^{c}$. No entanto, o mais interessante desta perturbação é que $W^{s}(p,(h \circ f))$ coincide com $W^{u}(p,(h \circ f))$ num intervalo $I \ni z_{0}$, e $I \subset U$. 
Assim, repassamos $f$ a $h \circ f$.

Seja $a>0$ tal que $\phi^{-1}([-2 a, 2 a]) \subset I$, onde $[-2 a, 2 a]=\{(x, 0)$, tal que $x \in[-2 a, 2 a]\}$.

Dado $\varepsilon>0$, para todo $\delta>0$, vamos produzir uma $\delta-C^{1}$ perturbação $g \in \operatorname{Diff}_{\omega}^{1}(M)$ de $f$, tal que $g(z)=f(z)$ para $z \notin f^{-1}(U)$, e $g$ tenha um conjunto básico hiperbólico $\Lambda \subset \overline{H(p, g)}$ $\operatorname{com} h(g \mid \Lambda)>\log |\lambda(p)|-\varepsilon$.

Consideremos uma vizinhança $\mathcal{N}$ de $f$, e seja $\delta>0$, pequeno o suficiente, tal que qualquer $g, \delta-C^{1}$ próximo de $f$, esteja em $\mathcal{N}$. Seja $N$ um inteiro positivo grande, e $A$ uma constante pequena dependendo de $N$, tal que $A N \rightarrow 0$. Consideremos a seguinte transformação que preserva área:

$$
\Phi(x, y)=\left(x, y+A \cos \frac{\pi x N}{2 a}\right) .
$$

$\Phi$ leva o segmento de reta $\{(x, 0) ;-a \leq x \leq a\}$ sobre a curva $J^{\prime}$; onde $J^{\prime}$ é o gráfico da função $x \mapsto A \cos \left(\frac{\pi N x}{2 a}\right), \operatorname{com}-a \leq x \leq a$. Assim, a altura máxima de $J^{\prime}$ é $A$, a menor é $-A$, e $J^{\prime}$ tem $N$ interseções com $J$. Figura 3.3. Denotemos por $I^{\prime}$ o gráfico $\phi^{-1}\left(J^{\prime}\right)$.

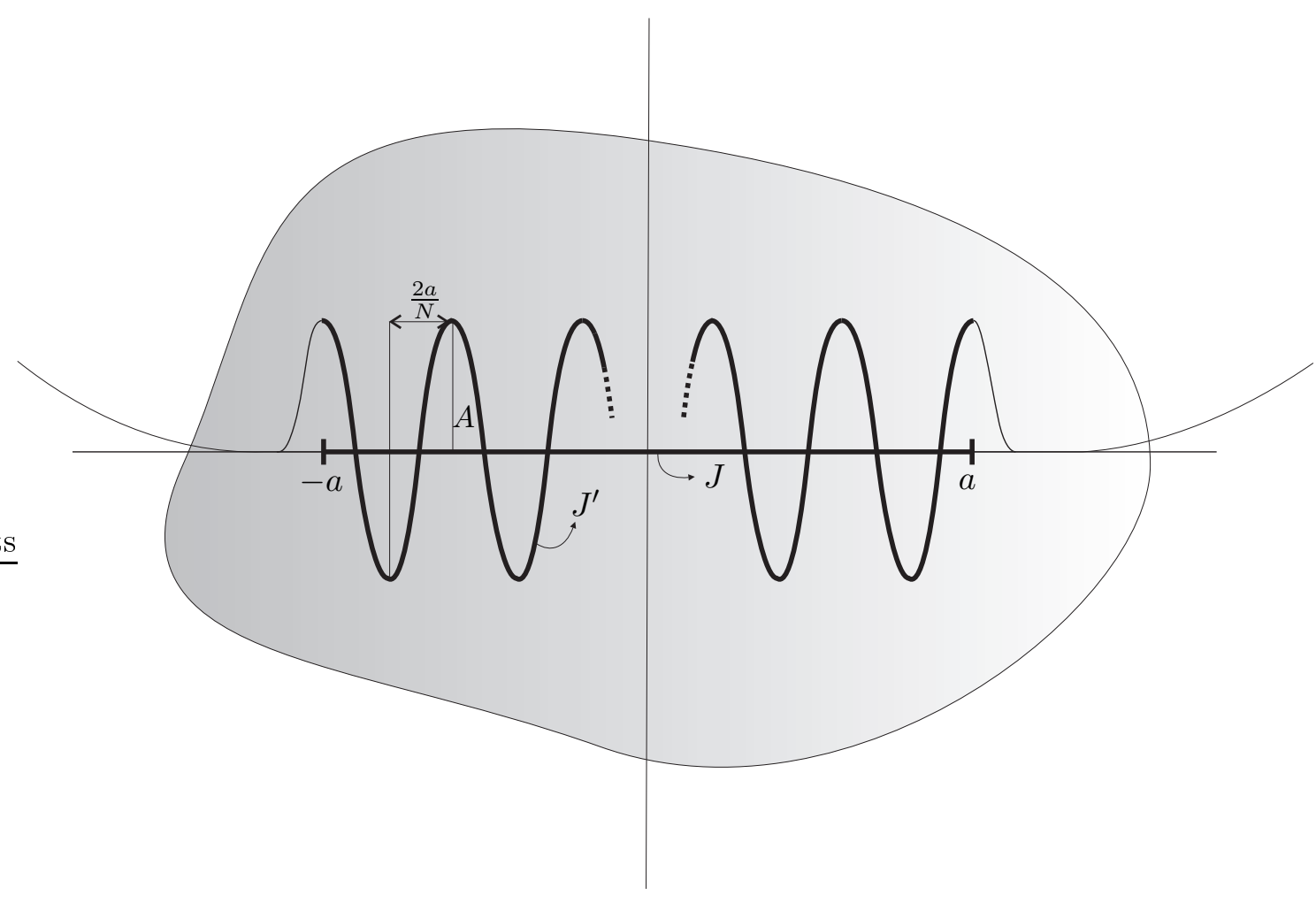

Figura 3.3:

Como

$$
D \Phi(x, y)=\left(\begin{array}{cc}
1 & 0 \\
-A \frac{\pi N}{2 a} \operatorname{sen} \frac{\pi x N}{2 a} & 1
\end{array}\right)
$$


para que $\Phi$ seja $\gamma-C^{1}$ próximo da $I d$, precisamos que $A \frac{\pi N}{2 a}<\gamma$. Assim, pelo Lema de Perturbação 2.2.2 existe constante $K_{1}>0$, tal que tomando $A=\frac{a \delta K_{1}}{2 \pi N}$, para algum $\delta>0$, existe difeomorfismo simplético $h \delta-C^{1}$ próximo da $I d, \operatorname{com} h=\Phi$, perto da origem, e $h=I d$ fora de uma vizinhança da origem.

Assim, usando a coordenada simplética $\phi$, podemos fazer esta perturbação, como feito anteriormente, na vizinhança $U$ de $z_{0}$. Consideremos, então, $g=h \circ f$. Deta forma $g$ é $\delta-C^{1}$ próximo de $f, g(z)=f(z)$ para $z \notin f^{-1}(U), I$ ainda pertence a $W^{s}(p, g)$, e $I^{\prime} \subset W^{u}(p, g)$.

Seja $W_{l o c}^{s}(p, g)$ um intervalo fechado em $W^{s}(p, g)$ contendo $I$ em seu interior, e seja $V$ uma vizinhança tubular de $W_{l o c}^{s}(p, g)$. Assumimos que $U$ esteja contido em $V$. Seja $\gamma_{1}$ a curva em $U$ dada por $x \equiv-a, 0 \leq y \leq 2 A$, e seja $\gamma_{2}$ a curva dada por $x \equiv a, 0 \leq y \leq 2 A$. Seja $\left\{z_{1}\right\}=\gamma_{1} \cap I^{\prime}$ e $\left\{z_{2}\right\}=\gamma_{2} \cap I^{\prime}$. Figura 3.4.

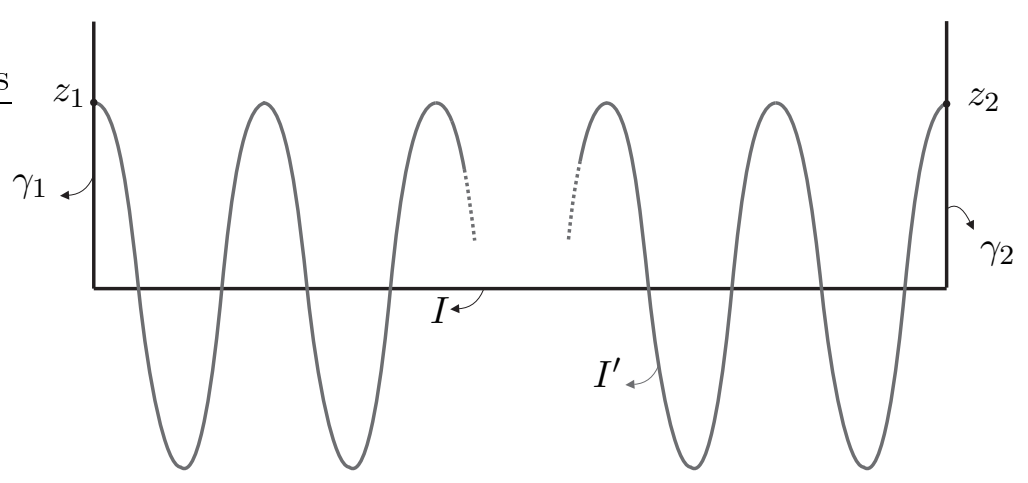

Figura 3.4:

Como $I^{\prime} \subset W^{u}(p, g)$, usando lambda Lema 1.1.15, iterados passados de $\gamma_{1}$ e $\gamma_{2}$ vão se acumular sobre $W_{l o c}^{s}(p, g)$.

Poderíamos assumir via uma aproximação preliminar que $f$ é $C^{2}$, [8]. Então, $g$ também é $C^{2}$, e assim, pelo Teorema de Hartman-Grobman 1.1.12, $C^{1}$ linearizavel sobre $W^{s}(p, g)$ e $W^{u}(p, g)$, perto do ponto $p$. Desta forma, existem constantes $K_{2}, K_{3}>0$ tal que se $g^{j}(z) \in V$ para $0 \leq j \leq m$, onde $m$ é o maior possível,

$$
K_{2}|\lambda(p)|^{-m} \leq d\left(z, W_{l o c}^{s}(p, g)\right) \leq K_{3}|\lambda(p)|^{-m},
$$

e se $g^{-j}(z) \in V$, para $0 \leq j \leq m$, então

$$
K_{2}|\lambda(p)|^{-m} \leq d\left(z, C\left(p, W^{u}(p, g) \cap V\right) \leq K_{3}|\lambda(p)|^{-m}\right.
$$

Para $n$ grande, as curvas $\gamma_{1}, \gamma_{2}, C\left(g^{-n}\left(z_{1}\right), g^{-n}\left(\gamma_{1}\right) \cap V\right)$ e $C\left(g^{-n}\left(z_{2}\right), g^{-n}\left(\gamma_{2}\right) \cap V\right)$ limitam um retângulo, $D_{n}$, em $U$ próximo de $I$. Consideremos , $\gamma_{n 1}^{\prime}$ e $\gamma_{n 2}^{\prime}$ os pedaços de $\gamma_{1}$ e $\gamma_{2}$ em $D_{n}$, respectivamente. 
Seja $n$ o menor inteiro positivo tal que $C\left(g^{-n}\left(z_{1}\right), g^{-n}\left(\gamma_{1}\right) \cap V\right)$ e $C\left(g^{-n}\left(z_{2}\right), g^{-n}\left(\gamma_{2}\right) \cap V\right)$, $g^{n}\left(\gamma_{n 1}^{\prime}\right)$ e $g^{n}\left(\gamma_{n 2}^{\prime}\right)$, estão $A / 4-C^{1}$ próximos de $W_{l o c}^{s}(p, g)$ e $I^{\prime}$, respectivamente. Para tal $n$, denotemos $D_{n}, \gamma_{n 1}^{\prime}$ e $\gamma_{n 2}^{\prime}$ por $D_{A}, \gamma_{1}^{\prime}$ e $\gamma_{2}^{\prime}$, respectivamente. Figura 3.5

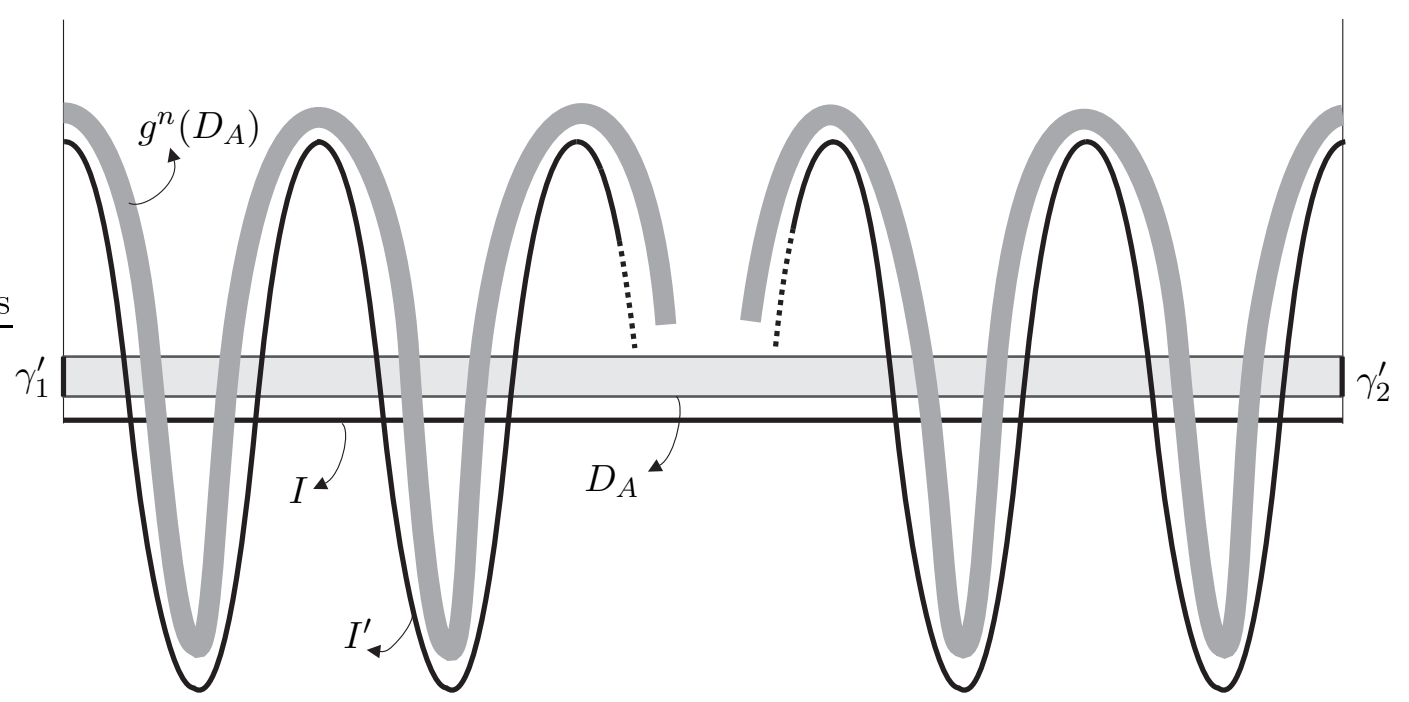

Figura 3.5:

Afirmação: Existem constantes $K_{4}, K_{5}>0$, independentes de $N$, tal que

$$
K_{4}|\lambda(p)|^{-n} \leq A \leq K_{5}|\lambda(p)|^{-n}
$$

Como $z_{1}$ e $z_{2} \in W^{u}(p, g)$ temos

$$
d\left(g^{-m}\left(z_{1}\right), g^{-m}\left(z_{2}\right)\right)=K|\lambda(p)|^{-m}, \text { para } m \text { grande }
$$

onde $K$ é uma constante, que depende do tempo, que $z_{1}$ e $z_{2}$ levam para entrar, definitivamente, na vizinhança $V$ ( olhando, neste caso, para os iterados passados de $g$ ). Notemos que $K$ não depende de $m$, para este suficientemente grande.

Pela escolha de $n$, temos que $d\left(g^{-n}\left(z_{1}\right), p\right), d\left(g^{-n}\left(z_{2}\right), p\right)<A / 4$, e assim

$$
K|\lambda(p)|^{-n}=d\left(g^{-n}\left(z_{1}\right), g^{-n}\left(z_{2}\right)\right)<A / 4 .
$$

Tomando $K_{4}=4 K$ temos

$$
A \geq K_{4}|\lambda(p)|^{-n}
$$

Sem perda de generalidade, podemos supor que $n$ seja o menor tal que $D_{A}$ esteja $A / 4$ próximo de $W_{l o c}^{s}(p, g)$. Por escolha deste $n$, deve existir $z \in D_{A}$ tal que

$$
d\left(g(z), W_{l o c}^{s}(p, g)\right) \geq A / 4 .
$$


No entanto, reduzindo $A$ se necessário, $z$ pode ser escolhido tal que $g(z)$ ainda esteja em $V$. Também, como $g^{n}\left(D_{A}\right)$ está $A / 4-C^{1}$ próximo de $I^{\prime}$, temos que $g^{n}\left(D_{A}\right) \subset V, \operatorname{logo} g^{n}(z) \in V$. Usando a desigualdade 3.2 no ponto $g(z)$, para $g^{n-1}$ e $m=1$, temos

$$
d\left(g(z), W_{l o c}^{s}\left(p, g^{n-1}\right)\right) \leq K_{3}|\lambda(p)|^{-(n-1)} .
$$

Com isto, mais a desigualdade 3.5 , e tomando $K_{5}=4 K_{3}|\lambda(p)|$,

$$
A \leq K_{5}|\lambda(p)|^{-n}
$$

Das equações 3.4 e 3.6, temos a afirmação.

Olhando para $g^{n}$ e $D_{A}$, temos uma ferradura de $N$ pernas. Assim, se $\Lambda_{1}$ é o conjunto maximal invariante para $g^{n} \mid D_{A}$, então $\Lambda_{1}$ é hiperbólico para $g^{n}$ e $g^{n} \mid \Lambda_{1}$ é topologicamente conjugado ao shift agindo no espaço das sequências de $N$ símbolos, $\Sigma_{N}$. Logo, pelas Proposições 1.1 .23 e 1.1 .25 , temos que $h\left(g^{n} \mid \Lambda_{1}\right)=\log N$.

Tomando

$$
\Lambda=\bigcup_{0 \leq j \leq n} g^{j}\left(\Lambda_{1}\right)
$$

temos que $\Lambda$ é um conjunto hiperbólico para $g$, Exemplo 1.1.6. Usando a Proposição 1.1.22,

$$
h(g \mid \Lambda)=\frac{1}{n} \log N
$$

Notemos que da construção de $g$, juntamente com o fato de $\Lambda$ possuir pontos periódicos densos, Teorema 1.1.20, temos um ponto periódico em $\Lambda$ que é homoclinicamente relacionado com $p$. Assim, temos $\Lambda \subset \overline{H(p, g)}$.

Usando a Afirmação, mais a escolha de $A$, temos o seguinte:

$$
K_{4}|\lambda(p)|^{-n} \leq \frac{a \delta K_{1}}{2 \pi N} \leq K_{5}|\lambda(p)|^{-n}
$$

o que implica

$$
\frac{1}{n} \log K_{4}-\log |\lambda(p)| \leq \frac{1}{n} \log \frac{a \delta K_{1}}{2 \pi}-\frac{1}{n} \log N \leq \frac{1}{n} \log K_{5}-\log |\lambda(p)| .
$$

Quando $N \rightarrow \infty$, implica que $A \rightarrow 0$, e assim, por escolha, $n \rightarrow \infty$. Agora, fazendo $N \rightarrow \infty$ na desigualdade acima, temos, pelo Teorema do Confronto, que

$$
\lim _{N \rightarrow \infty} \frac{1}{n} \log N=\log |\lambda(p)| \text {. }
$$

Podemos, então, escolher $N$ grande o suficiente, tal que $\frac{1}{n} \log N>\log |\lambda(p)|-\epsilon$. E assim, usando a igualdade (3.7)

$$
h(g \mid \Lambda)=\frac{1}{n} \log N>\log |\lambda(p)|-\epsilon .
$$

Para $\tau(p)>1$, a prova é análoga, exceto o fato que $z_{0} \in W^{s}(p, f) \cap W^{u}\left(f^{k}(p), f\right)$, para $0 \leq k<\tau(p)$. O $n$ escolhido na primeira parte pode ser escolhido da forma $n=\tau(p) n_{1}+k$. 
Seguindo um roteiro análogo ao feito na demonstração da afirmação, acima, conseguimos a seguinte estimativa

$$
K_{6}|\lambda(p)|^{-n_{1}} \leq A \leq K_{7}|\lambda(p)|^{-n_{1}} .
$$
que

Substituindo $A=\frac{a \delta K_{1}}{2 \pi N}$, e seguindo o mesmo raciocínio da primeira parte, concluímos

$$
\lim _{N \rightarrow \infty} \frac{1}{n} \log N=\frac{1}{\tau(p)} \log |\lambda(p)| .
$$

E, portanto, tomando $N$ grande o suficiente,

$$
h(g \mid \Lambda)=\frac{1}{n} \log N>\frac{1}{\tau(p)} \log |\lambda(p)|-\varepsilon .
$$

O que demontra a Parte $(a)$.

Parte (b)

Como na Parte (a), suponhamos $\tau(p)=1$. Consideremos o retângulo $D_{A}$, e a aplicação $g^{n}$.

As $N$ componentes de $g^{n}\left(D_{A}\right) \cap D_{A}$, são faixas inclinidas cruzando $D_{A}$, assim como mostra a Figura 3.6.

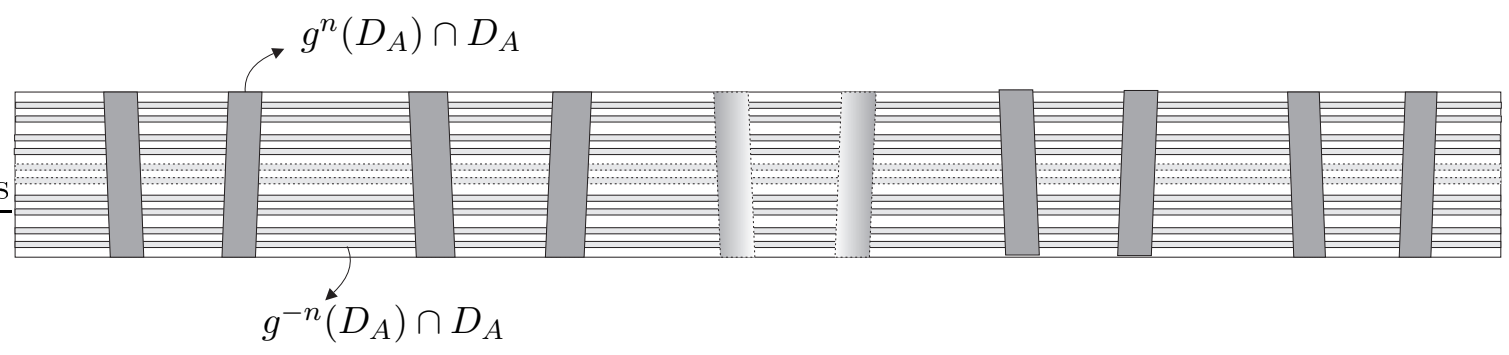

Figura 3.6:

Também, $g^{-n}\left(D_{A}\right) \cap D_{A}$ consiste de $N$ faixas cruzando horizontalmente $D_{A}$. Figura 3.6. Assim, temos que $g^{-n}\left(D_{A}\right) \cap g^{n}\left(D_{A}\right) \cap D_{A}$ contém $N^{2}$ quadriláteros disjuntos. Notemos ainda, que estes formam uma cobertura de $\Lambda_{1}$, e mais, retirando as fronteiras destes quadriláteros temos uma cobertura aberta de $\Lambda_{1}$. Assim,

$$
\bigcap_{0 \leq j \leq k} g^{j n} D_{A}
$$

consiste de $N^{k}$ faixas inclinadas, e

$$
\bigcap_{-k \leq j \leq 0} g^{j n} D_{A}
$$

de $N^{k}$ faixas horizontais. Logo, a interseção destas faixas nos dão $N^{2 k}$ discos em $D_{A}$. Além disto,

$$
\Lambda_{1} \subset \bigcap_{-k \leq j \leq k} g^{j n} D_{A}
$$


No entanto, o retângulo $g^{k n}\left(D_{A}\right)$ está contraindo na direção horizontal e esticando na direção vertical conforme $k$ cresce. Como estamos numa vizinhança pequena de $p$, esta taxa de contração é próxima de $|\lambda(p)|^{-n}$. Assim, podemos tomar uma constante $K_{8}$, independente de $N$, tal que para todo

$$
\begin{gathered}
U_{i} \in \bigcap_{-k \leq j \leq k} g^{j n} D_{A}, \\
\operatorname{diam}\left(U_{i}\right) \geq\left(K_{8}|\lambda(p)|^{-n}\right)^{k} .
\end{gathered}
$$

Desta forma,

$$
\begin{aligned}
& H_{\varepsilon}^{\beta}\left(\Lambda_{1}\right)=\inf \left\{\begin{array}{ll}
\sum\left(\operatorname{diam} U_{i}\right)^{\beta}: & \left\{U_{i}\right\} \text { é uma cobertura aberta de } \Lambda_{1} \\
& \text { e cada elemento possui diâmetro menor do que } \varepsilon
\end{array}\right\} . \\
& =\inf \left\{\sum\left(\operatorname{diam} U_{i}\right)^{\beta}, \quad U_{i} \in \bigcap_{-k \leq j \leq k} g^{j n} D_{A} \text {, para } k \text { suficientemente grande }\right\} \\
& \geq \inf \left\{\sum\left(K_{8}|\lambda(p)|^{-n}\right)^{k \beta}, \quad U_{i} \in \bigcap_{-k \leq j \leq k} g^{j n} D_{A} \text {, para } k \text { suficientemente grande }\right\} \text {, }
\end{aligned}
$$

Quando $\varepsilon \rightarrow 0$, temos que $k \rightarrow \infty$, nas desigualdades acima. Assim,

$$
H^{\beta}\left(\Lambda_{1}\right) \geq \liminf _{k \rightarrow \infty} N^{2 k}\left(K_{8}|\lambda(p)|^{-n}\right)^{k \beta} .
$$

Seja $\alpha=H D\left(\Lambda_{1}\right)=\inf \left\{\beta ; H^{\beta}\left(\Lambda_{1}\right)=0\right\}$. Tomando $\alpha_{1}=\inf \left\{\beta ; \liminf N^{2 k}\left(K_{8}|\lambda(p)|^{-n}\right)^{k \beta}=\right.$ $0\}$, pela desigualdade 3.8 , temos que $\alpha \geq \alpha_{1}$.

Agora, se $\beta$ é tal que $N^{2}\left(K_{8}|\lambda(p)|^{-n}\right)^{\beta}>1$. Então,

$$
\liminf _{k \rightarrow \infty} N^{2 k}\left(K_{8}|\lambda(p)|^{-n}\right)^{\beta k}=\infty .
$$

E se $\beta$ é tal que $N^{2}\left(K_{8}|\lambda(p)|^{-n}\right)^{\beta}<1$, temos que

$$
\liminf _{k \rightarrow \infty} N^{2 k}\left(K_{8}|\lambda(p)|^{-n}\right)^{\beta k}=0 .
$$

Desta forma, $\alpha_{1}$ é tal que $N^{2}\left(K_{8}|\lambda(p)|^{-n}\right)^{\alpha_{1}}=1$. Ou seja,

$$
\alpha_{1}=\frac{2 \log N}{n \log |\lambda(p)|-\log K_{8}} .
$$

Usando a estimativa da Afirmação feita na parte (a), temos que

$$
\frac{a \delta K_{1}}{2 \pi N} \leq K_{5}|\lambda(p)|^{-n} \Longrightarrow \frac{2 K_{5} \pi}{a \delta K_{1}} N \geq|\lambda(p)|^{n} .
$$

Tomando $K_{9}>\frac{2 K_{5} \pi}{a \delta K_{1}}$

$$
n \log |\lambda(p)|<\log K_{9}+\log N .
$$

Por fim, usando esta desigualdade em 3.9, temos

$$
\alpha_{1}>\frac{2 \log N}{\log N-\log K_{8}+\log K_{9}} .
$$


Como esta desigualdade não depende de $N$, temos o seguinte

$$
\alpha_{1} \geq \lim _{N \rightarrow \infty} \frac{2 \log N}{\log N-\log K_{8}+\log K_{9}}=2 .
$$

No entanto, $M$ tem dimensão 2, logo

$$
2 \geq \alpha>\alpha_{1} \geq \frac{2 \log N}{\log N-\log K_{8}+\log K_{9}} \longrightarrow 2 .
$$

Assim, dado $\varepsilon>0$, podemos tomar $N$ grande o suficiente tal que

$$
H D\left(\Lambda_{1}\right)=\alpha>2-\varepsilon .
$$

Como $\Lambda_{1} \subset \Lambda$, temos que $H D(\Lambda)>2-\varepsilon$.

Agora, para $g_{1}$ próximo de $g$, cada componente de

$$
\bigcap_{-k \leq j \leq k} g_{1}^{j n} D_{A}
$$

tem diâmetro maior do que $\left(K_{8}|\lambda(p)|^{-n}-\gamma\right)^{k} \operatorname{com} \gamma>0$ pequeno. Então, repetindo as contas anteriores para $g_{1}$, temos que $H D\left(\Lambda\left(g_{1}\right)\right)>2-\varepsilon$, onde $\Lambda\left(g_{1}\right)$ é a continuação do conjunto hiperbólico $\Lambda$, Teorema 1.1.18.

O caso geral é inteiramente análogo, ocorrendo apenas uma mudança na constante de estimativa do diâmetro das faixas em questão.

Na Proposição 3.2.6 temos como hipótese uma tangência entre $W^{u}(o(p))$ e $W^{s}(o(p))$, onde $p$ é um ponto periódico hiperbólico de um difeomorfismo não Anosov $f$. No entanto, vamos mostrar que em $\mathcal{D}$, a menos de uma perturbação $C^{1}$, este fato acontece. Ou seja, no complementar dos difeomorfismos de Anosov, o conjunto dos difeomorfismos que exibem tangência homoclínicas são densos.

Definição 3.2.7. Se $L: E \rightarrow F$ é uma aplicação linear entre espaços lineares normados, definimos

$$
m(L)=\inf _{|v|=1}|L v|
$$

a conorma de $L$.

Sejam $L, T: E \rightarrow F$ aplicações lineares, como na definição acima. Uma propriedade interessante de conorma é que:

$$
m(L T) \geq m(L) m(T) .
$$

De fato,

$$
\begin{aligned}
m(L T) & =\inf _{|v|=1}|L(v) T(v)| \\
& \geq \inf _{|v|=1}|L(v)| \inf _{|v|=1}|T(v)| \\
& =m(L) m(T) .
\end{aligned}
$$


Denotemos por $\mathcal{H}$ o subconjunto residual $\mathcal{B}$ em $\operatorname{Diff}_{\omega}^{1}(M)$, dado pelo Teorema 3.2.5.

Dado um ponto $p$ periódico hiperbólico para $g \in \mathcal{H}$, e $q \in H(p, g)$, definimos $u(q, g)$ o menor inteiro positivo $n$ tal que

$$
m\left(D g^{n}(q) \mid \mathbb{E}_{q}^{u}\right) \geq 2
$$

onde $\mathbb{E}_{q}^{u}=T_{q} W^{u}(p, g)$, é a direção instável de $g$ no ponto $q$.

Lema 3.2.8. Seja $M$ uma variedade simplética compacta, conexa, de dimensão dois, $f \in \mathcal{D}$, e p um ponto periódico hiperbólico para $f$. Então, existem sequências $g_{i} \in \mathcal{H}, q_{i} \in H\left(p\left(g_{i}\right), g_{i}\right)$, com $g_{i} \rightarrow f$ em $\operatorname{Diff}_{\omega}^{1}(M), e$

$$
\max \left\{u\left(q_{i}, g_{i}\right), u\left(q_{i}, g_{i}^{-1}\right)\right\} \rightarrow \infty
$$

quando $i \rightarrow \infty$. Onde $p\left(g_{i}\right)$ é a continuação do ponto $p$, para $g_{i}$.

Demonstração. Suponhamos que exista uma vizinhança $\mathcal{U}$ de $f$ em $\operatorname{Diff}_{\omega}^{1}(M)$ e um inteiro positivo $\nu>0$ tal que

$$
\sup _{\substack{q \in H(p(g), g) \\ g \in \mathcal{H} \cap \mathcal{U}}}\left\{u(q, g), u\left(q, g^{-1}\right)\right\} \leq \nu .
$$

Mostremos que com estas condições $f$ é Anosov, e assim temos uma contradição.

Primeiro, vamos mostrar que $\overline{H(p, f)}$ é um conjunto hiperbólico.

Seja $K_{1}=\inf \left\{m\left(D f^{j}(z)\right) ; 0 \leq j \leq \nu, z \in M\right\}$. Da compacidade de $M$, continuidade de $m$, mais o fato de $f \in \operatorname{Diff}_{\omega}^{1}(M)$ temos que $K_{1}>0$. Sendo assim, podemos tomar uma vizinhança menor $\mathcal{U}_{1} \subset \mathcal{U}$ de $f$, se necessário, tal que

$$
\left|m\left(D g^{j}(z)\right)-m\left(D f^{j}(z)\right)\right|<\frac{K_{1}}{2}, \quad 0 \leq j \leq \nu, z \in M, g \in \mathcal{U}_{1} .
$$

Daí, para todo $z \in M$, e $0 \leq j \leq \nu$,

$$
\begin{aligned}
m\left(D g^{j}(z)\right) & >\frac{-K_{1}}{2}+m\left(D f^{j}(z)\right) \\
& \geq \frac{-K_{1}}{2}+\inf \left\{m\left(D f^{j}(z)\right) ; 0 \leq j \leq \nu, z \in M\right\} \\
& =\frac{K_{1}}{2} .
\end{aligned}
$$

Disto podemos tirar que

$$
\inf \left\{m\left(D g^{j}(z)\right) ; 0 \leq j \leq \nu, z \in M, g \in \mathcal{U}_{1}\right\} \geq \frac{K_{1}}{2} .
$$

Escolhemos $h>0$ tal que $2^{h} \frac{K_{1}}{2}>2$.

Afirmação 1: Existe $n_{1}>0$ tal que $m\left(D g^{n_{1}}(q) \mid \mathbb{E}_{q}^{u}\right)>2$ para todo $q \in H(p(g), g)$ e $g \in \mathcal{U}_{1} \cap \mathcal{H}$. 
Seja $q \in H(p(g), g)$. Por escolha de $\nu$, e definição de $u(q, g)$, existe inteiro $0<n(q) \leq \nu$ tal que

$$
m\left(D g^{n(q)}(q) \mid \mathbb{E}_{q}^{u}\right) \geq 2
$$

Como $o(q) \in H(p(g), g)$, tomemos a maior sequência de inteiros possível,

$$
0=m_{0}<m_{1}<m_{2}<\ldots<m_{k}<h \nu
$$

tal que $\left(m_{i}-m_{i-1}\right)=n\left(g^{m_{i-1}}(q)\right)$. Assim, $m_{i}-m_{i-1} \leq \nu$ e $h \nu-m_{k} \leq \nu ; \operatorname{logo} k \geq h$. Daí,

$$
\begin{aligned}
m\left(D g^{h \nu}(q) \mid \mathbb{E}_{q}^{u}\right) & \geq m\left(D g^{h \nu-m_{k}}\left(g^{m_{k}}(q)\right) \mid \mathbb{E}_{g^{m_{k}(q)}}^{u}\right) \ldots m\left(D g^{m_{1}}(q) \mid E_{q}^{u}\right) \\
& \geq m\left(D g^{h \nu-m_{k}}\left(g^{m_{k}}(q)\right) \mid \mathbb{E}_{g^{m_{k}(q)}}^{u}\right) 2^{k} \\
& \geq \frac{K_{1}}{2} 2^{h} \\
& >2
\end{aligned}
$$

onde foi usado Regra da Cadeia e a propriedade de conorma (3.10) na primeira desigualdade, a equação (3.12) na segunda desigualdade, e a equação (3.11) na terceira desigualdade.

Tomando $n_{1}=h \nu$,

$$
m\left(D g^{n_{1}}(q) \mid \mathbb{E}_{q}^{u}\right)>2 \text {, para todo } q \in H(p(g), g), g \in \mathcal{U}_{1} \cap \mathcal{H}
$$

O que prova a Afirmação 1.

Similarmente, existe um inteiro $n_{2}>0$ tal que $m\left(D g^{-n_{2}}(q) \mid \mathbb{E}_{q}^{s}\right)>2$, para $q \in H(p(g), g)$, $g \in \mathcal{U}_{1} \cap \mathcal{H}$.

Afirmação 1': $m\left(D g^{-n_{1}}(q) \mid \mathbb{E}_{q}^{u}\right)<1 / 2$ para todo $q \in H(p(g), g)$ e $g \in \mathcal{U}_{1} \cap \mathcal{H}$.

De fato, seja $v \in \mathbb{E}_{q}^{u}$ um vetor unitário qualquer, assim, pela Afirmação 1,

$$
\left|D g^{n_{1}}\left(g^{-n_{1}}(q)\right)\left(\frac{D g^{-n_{1}}(q)(v)}{\left|D g^{-n_{1}}(q)(v)\right|}\right)\right|>2,
$$

que implica,

$$
1=|v|>2\left|D g^{-n_{1}}(q)(v)\right| .
$$

E, portanto, $m\left(D g^{-n_{1}}(q) \mid \mathbb{E}_{q}^{u}\right)<1 / 2$

Seja $y \in \overline{H(p, f)}$ e considere duas sequências $\mathbb{E}_{q_{i}}^{u}$ e $\mathbb{E}_{q_{i}^{\prime}}^{u}$, com $g_{i}, g_{i}^{\prime} \in \mathcal{H} \cap \mathcal{U}_{1}, q_{i} \in H\left(p\left(g_{i}\right), g_{i}\right)$, $q_{i}^{\prime} \in H\left(p\left(g_{i}^{\prime}\right), g_{i}^{\prime}\right)$; tal que $q_{i}, q_{i}^{\prime} \rightarrow y$ e $g_{i}, g_{i}^{\prime} \rightarrow f$, quando $i \rightarrow \infty$.

Afirmação 2: Existe um subespaço $\mathbb{E}_{y}^{u} \subset T_{y} M$ tal que $\mathbb{E}_{q_{i}}^{u}, \mathbb{E}_{q_{i}^{\prime}}^{u} \rightarrow \mathbb{E}_{y}^{u}$, quando $i \rightarrow \infty$, num senso de Grassman.

Primeiro, definimos $\mathbb{E}_{y}^{u}$ como sendo o limite de uma subsequência de $\left\{\mathbb{E}_{q_{i}}^{u}\right\}$. Repassando, $\left\{\mathbb{E}_{q_{i}}^{u}\right\}$ para esta subsequência, podemos assumir que $\mathbb{E}_{q_{i}}^{u} \rightarrow \mathbb{E}_{y}^{u}$. 
Suponhamos por absurdo, que $\mathbb{E}_{q_{i}^{\prime}}^{u}$ não converge a $\mathbb{E}_{y}^{u}$. Isto é, existe $v \in T_{y} M,|v|=1$, tal que $v \notin \mathbb{E}_{y}^{u}$, e $v$ é limite subsequencial de $\mathbb{E}_{q_{i}^{\prime}}^{u}$.

Seja $n_{3} \geq \max \left\{n_{1}, n_{2}\right\}$. Como $v \notin \mathbb{E}_{y}^{u}$, para $i$ suficientemente grande $v \notin \mathbb{E}_{q_{i}}^{u}$. Assim, para $i$ grande, $v$ tem componente $v^{s}$ em $\mathbb{E}_{q_{i}}^{s}$, onde usamos o fato que $T_{q_{i}} M=\mathbb{E}_{q_{i}}^{s} \oplus \mathbb{E}_{q_{i}}^{u}$. Usando a Afirmação 1,

$$
\left|D g_{i}^{-n_{3}}\left(q_{i}\right)(v)\right| \geq\left|D g_{i}^{-n_{2}}\left(q_{i}\right)\left(v^{s}\right)\right|>2 .
$$

Por outro lado, para infinitos valores de $i, v$ é próximo de $\mathbb{E}_{q_{i}^{\prime}}^{u}$ E assim, pela Afirmação $1^{\prime},\left|D\left(g_{i}^{\prime}\right)^{-n_{3}}\left(q_{i}^{\prime}\right)(v)\right| \leq \frac{1}{2}$.

Agora, como $g_{i}, g_{i}^{\prime} \rightarrow f$, temos

$$
\frac{1}{2} \geq\left|D f^{-n_{3}}(y)(v)\right|>2,
$$

o que é absurdo. Logo $\mathbb{E}_{q_{i}^{\prime}}^{u}$ converge para $\mathbb{E}_{y}^{u}$. Mais ainda,

$$
m\left(D f^{n_{3}}(y) \mid \mathbb{E}_{y}^{u}\right)>2 .
$$

Similarmente, os subespaços $\mathbb{E}_{q_{i}}^{s}$ convergem ao subespaço $\mathbb{E}_{y}^{s} \subset T_{y} M$, e além disto $m\left(D f^{-n_{3}}(y) \mid \mathbb{E}_{y}^{s}\right)>2$.

Como $T_{q_{i}} M=\mathbb{E}_{q_{i}}^{u} \oplus \mathbb{E}_{q_{i}}^{s}$, temos diretamente que $T_{y} M=\mathbb{E}_{y}^{u} \oplus \mathbb{E}_{y}^{s}$.

De fato, seja $v \in T_{y} M$. Existem $v_{i} \in T_{q_{i}} M$ tal que $v_{i} \rightarrow v$. Para cada $v_{i}$, existe decomposição $v_{i}^{s} \in \mathbb{E}_{q_{i}}^{s}, v_{i}^{u} \in \mathbb{E}_{q_{i}}^{u}$, tal que $v_{i}=v_{i}^{s}+v_{i}^{u}$. Pela Afirmação 2, $v_{i}^{s}$ converge para algum $v^{s} \in \mathbb{E}_{y}^{s}$, e anologamente $v_{i}^{u}$ converge para algum $v_{u} \in \mathbb{E}_{y}^{u}$. Da unicidade do limite temos $v=v^{s}+v^{u}$, como queríamos.

Agora, sabemos que $D g_{i}\left(q_{i}\right) \mid \mathbb{E}_{q_{i}}^{u}=\mathbb{E}_{g_{i}\left(q_{i}\right)}^{u}$, para todo $i$. Como $q_{i} \rightarrow y$, pela Afirmação 2, e por $g_{i}$ convergir para $f$, na topologia $C^{1}, D f(y) \mid \mathbb{E}_{y}^{u}=\mathbb{E}_{f(y)}^{u}$. Desta forma temos que $\left\{\mathbb{E}_{y}^{u}\right\}$ é sub-fibrado invariante de $T M$. Analogamente, $\left\{\mathbb{E}_{y}^{s}\right\}$ também é sub-fibrado invariante.

Da demonstração da Afirmação 2, temos que:

$$
m\left(D f^{n_{3}}(y) \mid \mathbb{E}_{y}^{u}\right)>2 \quad \text { e } \quad m\left(D f^{-n_{3}}(y) \mid \mathbb{E}_{y}^{s}\right)>2 \text {, para todo } y \in \overline{H(p, f)} .
$$

Seja $c_{1}=\inf \left\{m\left(D f^{j}(y) \mid \mathbb{E}_{y}^{u}\right) ; y \in \overline{H(p, f)}, 0 \leq j \leq n_{3}\right\}$ e escolhemos $2>\beta_{1}>1$ tal que $\beta_{1}^{(k+1) n_{3}}<2^{k}$, para todo $k \geq 0$.

Fixemos $n \geq 0$. Se $n \leq n_{3}$,

$$
m\left(D f^{n}(y) \mid \mathbb{E}_{y}^{u}\right) \geq c_{1}>\frac{c_{1}}{2} \beta_{1}^{n} .
$$

Se $n \geq n_{3}$, escrevemos $n=k n_{3}+r$, com $k>0$ e $0 \leq r<n_{3}$. Assim,

$$
\begin{aligned}
m\left(D f^{n}(y) \mid \mathbb{E}_{y}^{u}\right) \geq & m\left(D f^{r}\left(f^{k n_{3}}(y)\right) \mid \mathbb{E}_{f^{k n_{3}(y)}}^{u}\right) m\left(D f^{n_{3}}\left(f^{(k-1) n_{3}}(y)\right) \mid \mathbb{E}_{f^{(k-1) n_{3}(y)}}^{u}\right) \\
& \ldots m\left(D f^{n_{3}}(y) \mid \mathbb{E}_{y}^{u}\right) \\
\geq & c_{1} 2^{k} \\
\geq & c_{1} \beta_{1}^{(k+1) n_{3}} \\
> & \frac{c_{1}}{2} \beta_{1}^{n} .
\end{aligned}
$$


Similarmente, existem constantes $c_{2}>0, \beta_{2}>1 \mathrm{com}$

$$
\left|D f^{n}(y)\right| \mathbb{E}_{y}^{s} \mid<c_{2} \beta_{2}^{-n}, \text { para } n \geq 0 .
$$

E assim, temos que $\overline{H(p, f)}$ é um conjunto hiperbólico para $f$.

Agora, mostremos que $\overline{H(p, f)}$ possui estrutura de produto local, isto é, para $\varepsilon>0$ pequeno e $x, y \in \overline{H(p, f)}$, temos que $W_{\varepsilon}^{u}(x) \cap W_{\varepsilon}^{s}(y) \subset \overline{H(p, f)}$.

Como $\overline{H(p, f)}$ é hiperbólico e compacto, podemos escolher $\varepsilon>0$ tal que se $d(x, y)<2 \varepsilon$, então $W_{\varepsilon}^{u}(x) \cap W_{\varepsilon}^{s}(y) \neq \emptyset$ e $W_{\varepsilon}^{u}(x)$ é transversal a $W_{\varepsilon}^{s}(y)$, onde $d$ é a métrica induzida em $\overline{H(p, f)}$. Da mesma forma, tomamos $0<\delta<\varepsilon$ tal que se $W_{\delta}^{u}(x) \cap W_{\delta}^{s}(y) \neq \emptyset$, então $d(x, y)<2 \epsilon$.

Sejam $x, y \in \overline{H(p, f)}$. Afirmamos que $W_{\delta}^{u}(x) \cap W_{\delta}^{s}(y) \in \overline{H(p, f)}$.

Por vacuidade, podemos assumir que $W_{\delta}^{u}(x) \cap W_{\delta}^{s}(y) \neq \emptyset$, e assim, $d(x, y)<2 \varepsilon$. Seja $z \in W_{\delta}^{u}(x) \cap W_{\delta}^{s}(y)$. Existem pontos $x_{1}, y_{1} \in H(p, f)$, tais que $x_{1}$ é próximo de $x$ e $y_{1}$ é próximo de $y$. Usando o Teorema 1.1.20, existem pontos periódicos hiperbólicos $p_{1}$ e $q_{1}$ de $f$ com $p_{1}$ próximo de $x, q_{1}$ próximo de $y$, e

(a) $W_{\delta}^{u}\left(p_{1}\right) \cap W_{\delta}^{s}\left(q_{1}\right) \neq \emptyset$,

(b) $W_{\delta}^{u}\left(p_{1}\right) \cap W_{\delta}^{s}\left(q_{1}\right)$ tem um ponto próximo de $z$.(Figura 3.7 )

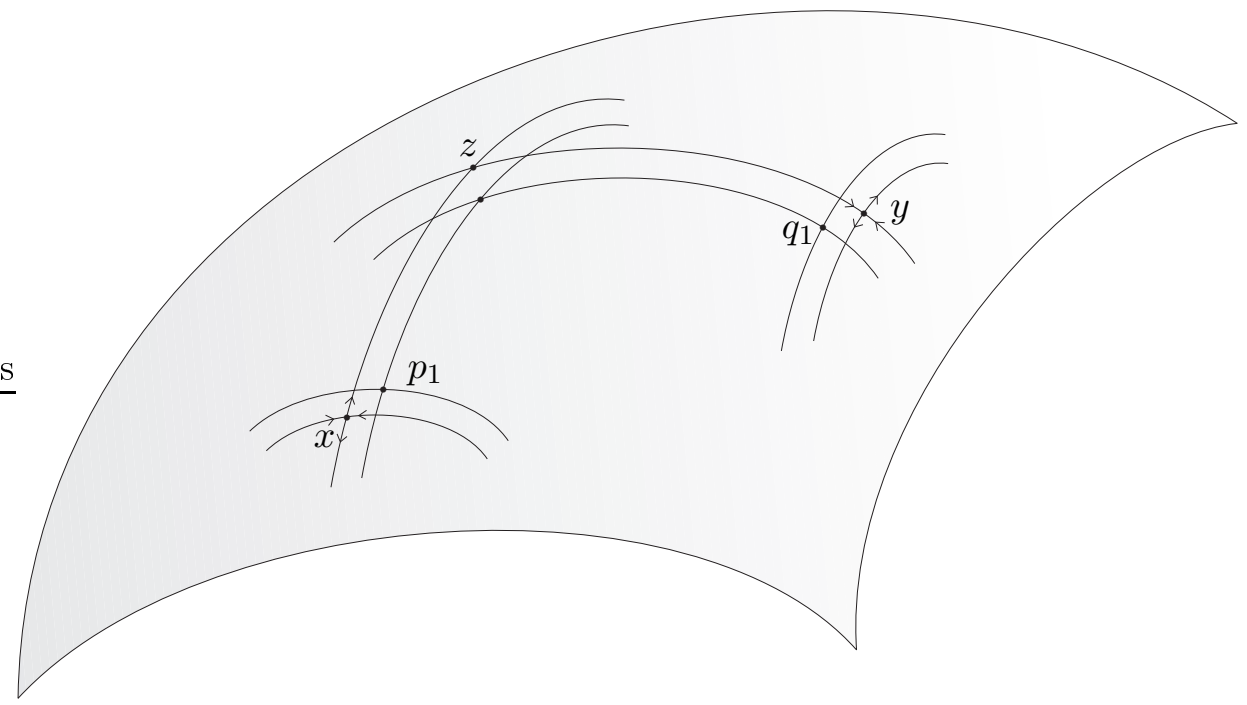

Figura 3.7:

Como $p_{1}$ e $q_{1}$ são homoclinicamente relacionados, usando lambda Lema 1.1.15, temos que $W_{\delta}^{u}\left(p_{1}\right) \cap W_{\delta}^{s}\left(q_{1}\right)$ é acumulado por pontos homoclínicos de $p_{1}$. Mas, $p_{1}$ é homoclinicamente relacionado com $p, \operatorname{logo} \overline{H\left(p_{1}, f\right)}=\overline{H(p, f)}$. Agora, como podemos tomar $x_{1}$ e $p_{1}, y_{1}$ e $q_{1}$ tão próximos quanto se queira de $x$ e $y$, respectivamente; temos que $z \in \overline{H(p, f)}$. 
Para completarmos a prova, basta mostrarmos que $\overline{H(p, f)}=M$. De fato, isto vai nos dar a contradição desejada. No entanto, pela conexidade de $M$, precisamos apenas mostrar que $\overline{H(p, f)}$ é um subconjunto aberto de $M$.

Usando a estrutura de produto local, é suficiente mostrarmos que para cada $y \in \overline{H(p, f)}$, $W_{\delta}^{u}(y) \cup W_{\delta}^{s}(y) \subset \overline{H(p, f)}$, onde $\delta$ é como acima.

Assim, consideremos uma sequência $g_{i} \in \mathcal{U}_{1} \cap \mathcal{H}$, tal que $g_{i} \rightarrow f$, quando $i \rightarrow \infty$. Fixado $i \in \mathbb{N}$ qualquer, seja $y \in \overline{H\left(p\left(g_{i}\right), g_{i}\right)}$, logo existe sequência $x_{j} \in H\left(p\left(g_{i}\right), g_{i}\right)$ convergindo para $y$. Assim, $W_{\delta}^{u}\left(x_{j}, g_{i}\right)$ e $W_{\delta}^{s}\left(x_{j}, g_{i}\right)$ convergem para $W_{\delta}^{u}\left(y, g_{i}\right)$ e $W_{\delta}^{s}\left(y, g_{i}\right)$, respectivamente. No entanto, pelo Teorema 3.2.5, como $g_{i} \in \mathcal{H}$, para todo $x \in H\left(p\left(g_{i}\right), g_{i}\right)$, temos que $W_{\delta}^{u}\left(x, g_{i}\right) \cup W_{\delta}^{s}\left(x, g_{i}\right) \subset \overline{H\left(p\left(g_{i}\right), g_{i}\right)}$; logo, por continuidade, $W_{\delta}^{u}\left(y, g_{i}\right) \cup W_{\delta}^{s}\left(y, g_{i}\right) \subset \overline{H\left(p\left(g_{i}\right), g_{i}\right)}$. E assim, temos que

$$
W_{\delta}^{u}\left(y, g_{i}\right) \cup W_{\delta}^{s}\left(y, g_{i}\right) \subset \overline{H\left(p\left(g_{i}\right), g_{i}\right)} \text {, para todo } y \in \overline{H\left(p\left(g_{i}\right), g_{i}\right)} \text {, e } i \in \mathbb{N} .
$$

Agora, dado $y \in \overline{H(p, f)}$, como $g_{i} \rightarrow f$, na topologia $C^{1}$, existe $y_{i} \in \overline{H\left(p\left(g_{i}\right), g_{i}\right)}$ com $y_{i} \rightarrow y$. E pela parte anteriror, temos que $W_{\delta}^{u}(y) \cup W_{\delta}^{s}(y) \subset \overline{H(p, f)}$, para todo $y \in \overline{H(p, f)}$. Como queríamos.

Proposição 3.2.9. Seja $M$ uma variedade simplética compacta, conexa, de dimensão dois, e $f \in \mathcal{D}$. Dado $\mathcal{T} \subset \operatorname{Diff}_{\omega}^{1}(M)$, vizinhança de $f$, e p um ponto periódico hiperbólico para $f$, existe $g \in \mathcal{T}$ tal que $W^{u}(o(p(g)), g)$ tangencia $W^{s}(o(p(g)), g)$ em algum ponto, onde $p(g)$ é a continuação do ponto periódico hiperbólico $p$.

Demonstração. Usando o Lema 3.2.8, consideremos a sequência $g_{i} \rightarrow f$ em $\operatorname{Diff}_{\omega}^{1}(M) \cap \mathcal{H}$, e $p_{i} \in H\left(p\left(g_{i}\right), g_{i}\right)$, tal que $u\left(p_{i}, g_{i}\right) \rightarrow \infty$. O caso $u\left(p_{i}, g_{i}^{-1}\right)$ é similar.

Como $\omega$ é uma 2-forma diferenciavel, $C^{\infty}$, temos que $\omega(p)$ é uma aplicação bilinear contínua. Logo $\omega(p)$ é limitada, isto é, existe $K_{p}$ tal que

$$
\left|\omega(p)\left(v_{1}, v_{2}\right)\right| \leq K_{p}\left|v_{1}\right|\left|v_{2}\right|, \text { para } p \in M \text {, e todo } v_{1}, v_{2} \in T_{p} M .
$$

Como $M$ é compacto, temos que existe $c \in \mathbb{R}$ tal que

$$
\left|\omega(p)\left(v_{1}, v_{2}\right)\right| \leq c\left|v_{1}\right|\left|v_{2}\right|, p \in M, v_{1}, v_{2} \in T_{p} M
$$

Por escolha de $u\left(p_{i}, g_{i}\right)$, tomando $n_{i}=u\left(p_{i}, g_{i}\right)-1$, existe algum vetor unitário $v_{i} \in$ $\mathbb{E}^{u}\left(p_{i}, g_{i}\right)$ tal que $\left|D g^{n_{i}}\left(v_{i}\right)\right|<2$.

Agora, para $0<\varepsilon<1$, podemos escolher $0<\delta<\varepsilon$ tal que $\delta /(1-\delta)<K^{-1} \varepsilon$, onde $K$ é dado pelo Lema de Perturbação 2.2.2. Assim, podemos construir uma $\varepsilon-C^{1}$ perturbação $h$ da identidade $I d$, sobre vizinhanças dos pontos $p_{i}, g_{i}\left(p_{i}\right), \ldots, g_{i}^{n_{i}}\left(p_{i}\right)$, tal que tomando $g_{i 1}=g_{i} \circ h$ tenhamos o seguinte

(a) $g_{i}(y)=g_{i 1}(y)$ para $y$ fora de uma pequena vizinhança de $\left\{p_{i}, g_{i}\left(p_{i}\right), \ldots, g_{i}^{n_{i}}\left(p_{i}\right)\right\}$;

(b) $g_{i 1}^{j}\left(p_{i}\right)=g_{i}^{j}\left(p_{i}\right)$ para $0 \leq j \leq n_{i}$; 
(c) $p_{i} \in H\left(p\left(g_{i 1}\right), g_{i 1}\right)$;

(d) $v_{i} \in E^{u}\left(p_{i}, g_{i 1}\right)$;

(e) $\left|D g_{i 1}^{n_{i}}\left(p_{i}\right)\left(v_{i}\right)\right| \leq 2(1-\delta)^{n_{i}}$.

Notemos que $g_{i 1}$ é uma $\varepsilon-C^{1}$ perturbação de $g_{i}$, em $\operatorname{Diff}_{\omega}^{1}(M)$.

Afirmação: $\omega\left|W^{u}\left(p_{i}, g_{i 1}\right) \equiv 0 \equiv \omega\right| W^{s}\left(p_{i}, g_{i 1}\right)$.

De fato, se $a_{1}, a_{2} \in T_{z} W^{u}\left(p_{i}, g_{i 1}\right)$, para $z \in W^{u}\left(p_{i}, g_{i 1}\right)$, então $D g_{i 1}^{-n}(z)\left(a_{1}\right)$ e $D g_{i 1}^{-n}(z)\left(a_{2}\right)$ aproximam-se de zero quando $n$ cresce. No entanto, como $g_{i 1} \in \operatorname{Diff}_{\omega}^{1}(M)$,

$$
\omega(z)\left(a_{1}, a_{2}\right)=\omega\left(g_{i 1}^{-n}(z)\right)\left(D g_{i 1}^{-n}\left(a_{1}\right), D g_{i 1}^{-n}(z)\left(a_{2}\right)\right) \text {, para todo } n \text {. }
$$

Fazendo $n \rightarrow \infty$, temos que $\omega(z)\left(a_{1}, a_{2}\right)=0$. E, portanto, $\omega(z)=0$, para $z \in W^{u}\left(p_{i}, g_{i}\right)$. Logo, $\omega \mid W^{u}\left(p_{i}, g_{i 1}\right) \equiv 0$. Similarmente, temos que $\omega \mid W^{s}\left(p_{i}, g_{i 1}\right) \equiv 0$.

Como $\omega$ é não degenerada, existe vetor $v_{i}^{\prime} \in \mathbb{E}^{s}\left(p_{i}, g_{i 1}\right)$ tal que $\omega\left(p_{i}\right)\left(v_{i}, v_{i}^{\prime}\right)=1$. Usando (3.13), temos

$$
\begin{aligned}
1=\omega\left(p_{i}\right)\left(v_{i}, v_{i}^{\prime}\right) & =\omega\left(g_{i 1}^{n_{i}}\left(p_{i}\right)\right)\left(D g_{i 1}^{n_{i}}\left(p_{i}\right)\left(v_{i}\right), D g_{i 1}^{n_{i}}\left(p_{i}\right)\left(v_{i}^{\prime}\right)\right) \\
& \leq c\left|D g_{i 1}^{n_{i}}\left(v_{i}\right)\right|\left|D g_{i 1}^{n_{i}}\left(v_{i}^{\prime}\right)\right| \\
& <2 c(1-\delta)^{n_{i}}\left|D g_{i 1}^{n_{i}}\left(v_{i}^{\prime}\right)\right|
\end{aligned}
$$

daí,

$$
\left|D g_{i 1}^{n_{i}}\left(v_{i}^{\prime}\right)\right|>(2 c)^{-1}(1-\delta)^{-n_{i}}
$$

Usando, novamente, a parte (b) do Lema de Perturbação 2.2.2, podemos fazer outra $\varepsilon-C^{1}$ perturbação, em $\operatorname{Diff}_{\omega}^{1}(M)$, de $g_{i 1}$ para $g_{i 2}$, sobre uma pequena vizinhança de $g_{i 1}^{-1}\left(p_{i}\right)$, exigindo que

(a) $g_{i 2}\left(g_{i 1}^{-1}\left(p_{i}\right)\right)=p_{i}$

(b) $D g_{i 2}\left(g_{i 1}^{-1}\left(p_{i}\right)\right)\left(D g_{i 1}^{-1}\left(p_{i}\right)\left(v_{i}\right)\right)=\left(\alpha v_{i}+\beta v_{i}^{\prime}\right)$,

$\operatorname{com} \beta / \alpha>\varepsilon / 2$. Com isto, temos que $\left(\alpha v_{i}+\beta v_{i}^{\prime}\right) \in \mathbb{E}^{u}\left(p_{i}, g_{i 2}\right)$, e $v_{i}^{\prime}$ continua pertencendo a $\mathbb{E}^{s}\left(p_{i}, g_{i 2}\right)$. Agora, $D g_{i 2}^{n_{i}}\left(p_{i}\right)\left(\alpha v_{i}+\beta v_{i}^{\prime}\right)$ pode ser escrito em coordenadas $\left(\xi_{1}, \xi_{2}\right)$, tal que

$$
\xi_{1} \in \mathbb{E}^{u}\left(g_{i 1}^{n_{i}}\left(p_{i}\right), g_{i 1}\right) \text { e } \xi_{2} \in \mathbb{E}^{s}\left(g_{i 1}^{n_{i}}\left(p_{i}\right), g_{i 1}\right) .
$$

Ou seja,

$$
\begin{aligned}
D g_{i 2}^{n_{i}}\left(p_{i}\right)\left(\alpha v_{i}+\beta v_{i}^{\prime}\right) & =D g_{i 1}^{n_{i}}\left(p_{i}\right)\left(\alpha v_{i}+\beta v_{i}^{\prime}\right) \\
& =\alpha D g_{i 1}^{n_{i}}\left(p_{i}\right)\left(v_{i}\right)+\beta D g_{i 1}^{n_{i}}\left(p_{i}\right)\left(v_{i}^{\prime}\right)
\end{aligned}
$$


Assim, usando a condição (e) da primeira perturbação, mais (3.14), temos

$$
\frac{\left|\xi_{2}\right|}{\left|\xi_{1}\right|}=\frac{\left|\beta D g_{i 1}^{n_{i}}\left(p_{i}\right)\left(v_{i}^{\prime}\right)\right|}{\left|\alpha D g_{i 1}^{n_{i}}\left(p_{i}\right)\left(v_{i}\right)\right|}>\frac{\beta(2 c)^{-1}(1-\delta)^{-n_{i}}}{\alpha 2(1-\delta)^{n_{i}}}>\delta(8 c)^{-1}(1-\delta)^{-2 n_{i}}
$$

Fazendo $i \rightarrow \infty$, temos que $n_{i} \rightarrow \infty$, e assim $\left|\xi_{1} / \xi_{2}\right| \rightarrow 0$. Daí, como $\mathbb{E}^{s}\left(p_{i}, g_{i 2}\right)=\mathbb{E}^{s}\left(p_{i}, g_{i 1}\right)$, temos que o ângulo entre $\mathbb{E}^{u}\left(g_{i 2}^{n_{i}}\left(p_{i}\right), g_{i 2}\right)$ e $\mathbb{E}^{s}\left(g_{i 2}^{n_{i}}\left(p_{i}\right), g_{i 2}\right)$ está indo para zero quando $i \rightarrow \infty$.

Logo $W^{s}\left(p_{i}, g_{i 2}\right)$ e $W^{u}\left(p_{i}, g_{i 2}\right)$ são $C^{1}$ próximos em $g_{i 2}^{n_{i}}\left(p_{i}\right)$, para $i$ grande o suficiente.

Por um processo similar ao usado na demonstração da Proposição 3.2.6, podemos fazer uma $\varepsilon-C^{1}$ perturbação de $g_{i 2}$ para $g_{i 3}$, tal que $W^{s}\left(p_{i}, g_{i 3}\right)$ e $W^{u}\left(p_{i}, g_{i 3}\right)$ sejam tangentes em $g_{i 2}^{n_{i}}\left(p_{i}\right)$. E assim, como $g_{i} \rightarrow f$, para $i \rightarrow \infty$, podemos tomar $i$ suficientemente grande tal que $g=g_{i 3}$ seja $4 \varepsilon-C^{1}$ próximo de $f$.

Finalmente, vamos demonstrar o Teorema 3.2.1.

Demonstração. do Teorema 3.2.1

De acordo com a Observação 3.2.2, para demonstrarmos o Teorema precisamos apenas nos preocupar com os difeomorfismos $f \in \mathcal{D}$, isto é, os difeomorfismos não Anosov.

Para inteiros positivos $n$ e $m$, seja $B_{n, m}$ o conjunto dos difeomorfismos $f$ em $\mathcal{D}$ tal que existam $p \in H i p_{n} f$ e um conjunto básico hiperbólico $\Lambda \subset \overline{H(p, f)}$, satisfazendo $h(f \mid \Lambda)>$ $s_{n}(f)-\frac{1}{m}$. Também, seja $B_{n, m}^{\prime}$ o conjunto dos difeomorfismos $f$ em $\mathcal{D}$ tal que $H_{i p} f$ seja não vazio, e para cada $p \in H_{i p} f$, existe um conjunto básico hiperbólico $\Lambda \subset \overline{H(p, f)}$ tal que $H D(\Lambda)>2-\frac{1}{m}$.

Afirmação: $B_{n, m}$ e $B_{n, m}^{\prime}$ são conjuntos abertos e densos em $\mathcal{D}$.

O Teorema segue diretamente da Afirmação, tomando

$$
\mathcal{B}=\bigcap_{n, m}\left[\left(B_{n, m} \cap B_{n, m}^{\prime}\right) \cup \mathcal{A}\right]
$$

Mostremos, então, a Afirmação. Seja $f \in \mathcal{D}$, e sejam $n$ e $m$ inteiros positivos quaisquer. Usando o Teorema 3.2.3 e a Proposição 3.2.4, podemos tomar $f_{1}, C^{1}$ próxima de $f$, tal que esta tenha pontos periódicos hiperbólicos e elípticos densos em $M$. Como

$$
s_{n}\left(f_{1}\right)=\max \left\{\frac{1}{\tau(p)} \log |\lambda(p)|, \quad p \in \operatorname{Hip}_{n} f_{1}\right\}
$$

podemos escolher $p$ tal que

$$
\frac{1}{\tau(p)} \log |\lambda(p)| \geq s_{n}\left(f_{1}\right)-\frac{1}{4 m}
$$

Como $f_{1}$ tem pontos elípticos, temos que $f_{1} \in \mathcal{D}$. Usando a Proposição 3.2.9, podemos encontrar $f_{2} C^{1}$ próxima de $f_{1}$ com uma tangência entre $W^{s}\left(o\left(p\left(f_{2}\right)\right), f_{2}\right)$ e $W^{u}\left(o\left(p\left(f_{2}\right)\right), f_{2}\right)$, 
onde $p\left(f_{2}\right)$ é a continuação do ponto periódico hiperbólico $p$, para $f_{2}$. Como a perturbação é $C^{1}$ temos que $|\lambda(p)|$ é próximo de $\left|\lambda\left(p\left(f_{2}\right)\right)\right|$. Desta forma, podemos escolher $f_{2}$ tal que

$$
\frac{1}{\tau\left(p\left(f_{2}\right)\right)} \log \left|\lambda\left(p\left(f_{2}\right)\right)\right|>\frac{1}{\tau(p)} \log |\lambda(p)|-\frac{1}{4 m}
$$

Usando, agora, a Proposição 3.2.6, podemos encontrar $f_{3}, C^{1}$ próxima de $f_{2}$, tal que $f_{3}$ tenha um conjunto hiperbólico básico $\Lambda$, e

$$
h\left(f_{3} \mid \Lambda\right)>\frac{1}{\tau\left(p\left(f_{2}\right)\right)} \log \left|\lambda\left(p\left(f_{2}\right)\right)\right|-\frac{1}{4 m} .
$$

Sabendo que $s_{n}($.$) é contínua, Lema 3.1 .3$, como $f_{3}$ é próxima de $f_{1}$, se $f^{\prime}$ é próxima de $f_{3}$, então

$$
s_{n}\left(f^{\prime}\right)<s_{n}\left(f_{1}\right)+\frac{1}{4 m} .
$$

Também, $\Lambda\left(f^{\prime}\right)$ é topologicamente conjugado a $\Lambda$, onde $\Lambda\left(f^{\prime}\right)$ é a continuação de $\Lambda$, para perturbações $f^{\prime}$ de $f_{3}$, Teorema 1.1.18. E assim, usando que entropia é invariante por conjugação topológica, mais as equações (3.15), (3.16),(3.17) e (3.18), temos que:

$$
\begin{aligned}
h\left(f^{\prime}\right) & \geq h\left(f^{\prime} \mid \Lambda\left(f^{\prime}\right)\right) \\
& =h\left(f_{3} \mid \Lambda\right) \\
& >\frac{1}{\tau\left(p\left(f_{2}\right)\right)} \log \left|\lambda\left(p\left(f_{2}\right)\right)\right|-\frac{1}{4 m} \\
& >\frac{1}{\tau(p)} \log |\lambda(p)|-\frac{1}{2 m} \\
& \geq s_{n}\left(f_{1}\right)-\frac{3}{4 m} \\
& >s_{n}\left(f^{\prime}\right)-\frac{1}{m},
\end{aligned}
$$

para todo $f^{\prime}$ suficientemente próximo de $f_{3}$. $\operatorname{Logo} B_{n, m}$ é aberto e denso em $\mathcal{D}$.

Similarmente podemos usar o item $(b)$ da Proposição 3.2 .6 para provarmos que $B_{n, m}^{\prime}$ é aberto e denso em $D$.

Com isto demonstramos a Afirmação, e portanto o teorema.

\subsection{Difeomorfismos de Anosov}

Nesta seção, vamos mostrar que a desigualdade (3.1) falha para um subconjunto aberto e denso, dentro dos difeomorfismos de Anosov. Para isto, consideramos a função $\phi^{u}$ definida na Seção 1.2.2. Como estamos no mundo simplético, podemos considerar $f$ de classe $C^{2}$, a menos de uma perturbação [8]. E isto é essencial, pois os resultados que vamos usar exigem classe de difereciabilidade $C^{2}$.

Podemos ver em [6], que no caso Anosov a medida de Lebesgue $m$ é o único estado de equilíbrio para o observável $\phi^{u}$. Agora, seja $\mu$ a única medida invariante que maximiza 
entropia, ou seja, o único estado de equilíbrio para o funcional $\psi \equiv 0$. Por [7], sabemos que esta medida tem uma estrutura geométrica interessante. Tomando $H_{n}$ como sendo o conjunto dos pontos periódicos de período $n$, para $f$, sabemos que:

$$
\mu_{n}=\frac{\sum_{p \in H_{n}} \delta_{p}}{\sharp H_{n}} \rightarrow \mu,
$$

quando $n \rightarrow \infty$, na topologia fraca*. Isto é,

$$
\int \phi d \mu_{n} \rightarrow \int \phi d \mu, \text { para todo } \phi \in C(M)
$$

Tomando $H_{n}^{\prime} \subset H_{n}$, tal que $H_{n}^{\prime}$ contenha apenas um representante para cada órbita periódica de período $n$, temos que $\sharp H_{n}=n \sharp H_{n}^{\prime}$. Agora,

$$
\begin{aligned}
-\int \phi^{u} d \mu_{n} & =\frac{\sum_{p \in H_{n}} \log \bar{\lambda}(p)}{\sharp H_{n}} \\
& =\frac{\sum_{p \in H_{n}^{\prime}} \frac{1}{n} \log |\lambda(p)|}{\sharp H_{n}^{\prime}} \\
& \leq s_{n}(f),
\end{aligned}
$$

onde usamos que $\log |\lambda(p)|=\sum_{i=1}^{n-1} \log \bar{\lambda}\left(f^{i}(p)\right)$, na segunda desigualdade.

Daí, fazendo $n \rightarrow \infty$, temos:

$$
-\int \phi^{u} d \mu \leq s(f)
$$

Pelo Teorema 1.2.5, temos que $m$ e $\mu$ são ergódicas. Sendo assim, ou elas são equivalentes ou são mutuamente singulares.

Suponhamos que exista constante $k$ tal que

$$
S_{m} \phi^{u}(x)-S_{m} \psi(x)=k m, \text { para todo } x \text { tal que } f^{m}(x)=x \text {, e } m \geq 0,
$$

onde $\psi \equiv 0$. Podemos, escrever a expressão acima da seguinte forma:

$$
\frac{1}{m} \log |\lambda(x)|=k, \text { para todo } x \text { tal que } f^{m}(x)=x, \text { e } m \geq 0 .
$$

Usando o item (b) do Lema de Perturbação 2.2.2, podemos perturbar $f$, tal que

$$
\frac{1}{m} \log |\lambda(x)| \neq k \text {, para algum ponto periódico } x \text {, de período } m \text {. }
$$

Assim, pela Proposição 1.2.6, temos que $m$ e $\mu$ são mutuamente singulares.

Usando o fato que toda variedade estável e instável é densa em $M$, temos que todos os pontos periódicos hiperbólicos são homoclinicamente relacionados. Assim, temos que $M$ é o único conjunto básico hiperbólico. Daí, usando o Teorema 1.2.7, temos que $P_{f}\left(\phi^{u}\right)=0$. 
Juntando todos os resultados apresentados, logo acima, temos:

$$
\begin{aligned}
0=P_{f}\left(\phi^{u}\right) & =h_{m}(f)+\int \phi^{u} d m \\
& >h_{\mu}(f)+\int \phi^{u} d \mu \\
& =h(f)+\int \phi^{u} d \mu .
\end{aligned}
$$

Daí,

$$
\begin{aligned}
h(f) & <-\int \phi^{u} d \mu \\
& \leq s(f) .
\end{aligned}
$$

Agora, pelo Teorema 1.1.23 e Corolário 3.1.3,temos que $h(f)<s(f)$ é uma condição $C^{1}$ aberta para difeomorfismos de Anosov. Logo a desigualdade (3.1) falha para um subconjunto aberto e denso de difeomorfismos dentro dos Anosov. 


\section{Propriedades topológicas de}

Estamos interessados em propriedades genéricas sobre $\operatorname{Diff}_{\omega}^{1}(M)$. Isto é, propriedades que são válidas para um subconjunto residual em $\operatorname{Diff}_{\omega}^{1}(M)$, onde um subconjunto residual é uma interseção enumerável de subconjuntos abertos e densos. No entanto, subconjuntos residuais são interessantes quando estamos sobre espaços de Baire. Isto é, em espaços de Baire, conjuntos residuais ainda são densos.

\section{A.1 $\operatorname{Diff}_{\omega}^{1}(M)$ é um espaço de Baire}

Um resultado muito usado na hora de reconhecer espaços de Baire, é o seguinte:

Proposição A.1.1. Todo espaço métrico completo $X$ é um espaço de Baire.

A demonstração deste resultado pode ser encontrada em [14].

Consideremos agora o espaço $C^{r}\left(M, \mathbb{R}^{s}\right)$ das aplicações diferenciáveis de classe $C^{r}, 0 \leq$ $r<\infty$, definidas sobre a variedade $M$. Temos em $C^{r}\left(M, \mathbb{R}^{s}\right)$ uma estrutura natural de espaço vetorial: $(f+g)(p)=f(p)+g(p)$, e $(\lambda f)(p)=\lambda f(p)$ para $f, g \in C^{r}\left(M, \mathbb{R}^{s}\right)$ e $\lambda \in \mathbb{R}$.

Podemos tomar um atlas $\left(\phi_{i}, U_{i}\right)$, tal que $\phi_{i}: B(2) \rightarrow U_{i}$ seja um difeomorfismo, onde $B(2)$ é a bola de centro 0 e raio 2. Tomemos em $M$ uma cobertura finita por abertos $V_{1}, \ldots, V_{k}$, tal que cada $V_{i}$ esteja contido no domínio de uma carta local $\left(\phi_{i}, U_{i}\right)$, e $\phi_{i}\left(V_{i}\right)=B(1)$. 
Para $f \in C^{r}\left(M, \mathbb{R}^{s}\right)$ denotemos por $f^{i}=f \circ \phi_{i}: B(2) \rightarrow \mathbb{R}^{s}$. Definimos

$$
\|f\|_{r}=\max _{i} \sup _{u \in B(1)}\left\{\left\|f^{i}(u)\right\|,\left\|D f^{i}(u)\right\|, \ldots,\left\|D^{r} f^{i}(u)\right\|\right\}
$$

Proposição A.1.2. $\|\cdot\|_{r}$ é uma norma completa em $C^{r}\left(M, \mathbb{R}^{s}\right)$.

Demonstração. É imediato que $\|\cdot\|_{r}$ é uma norma em $C^{r}\left(M, \mathbb{R}^{s}\right)$. Resta mostrarmos que toda sequência de cauchy é convergente.

Seja $f_{n}: M \rightarrow \mathbb{R}^{s}$ uma sequência de cauchy na norma $\|\cdot\|_{r}$. Se $p \in M, p \in V_{i}$ para algum $i$, e assim, dado $\varepsilon>0$ existe $n_{0}$ tal que

$$
\left\|f_{n}^{i}(u)-f_{m}^{i}(u)\right\|<\varepsilon, n, m \geq n_{0}, \phi_{i}(u)=p .
$$

Logo, $f_{n}(p)$ é uma sequência de cauchy em $\mathbb{R}^{s}$, e portanto, convergente.

Tomemos $f(p)=\lim f_{n}(p)$. Em particular, $f_{n}^{i}(u) \rightarrow f^{i}(u)$, para $u \in B(1)$ e $i=1, \ldots, k$. Por outro lado, para cada $u \in B(1), D f_{n}^{i}(u)$ é uma sequência de cauchy em $L\left(\mathbb{R}^{m}, \mathbb{R}^{s}\right)$, espaço das transformações lineares de $\mathbb{R}^{m}$ em $\mathbb{R}^{s}$. E assim, converge para uma transformação linear $T^{i}(u)$.

Afirmação: $D f_{n}^{i}$ converge uniformemente para $T^{i}$.

Como $D f_{n}^{i}$ é sequência de cauchy, existe $n_{0}$ tal que

$$
\left\|D f_{n}^{i}(u)-D f_{m}^{i}(u)\right\|<\frac{\varepsilon}{2}, \text { para } n, m \geq n_{0} .
$$

Agora, dado $u \in B(1)$ existe $n_{u}$ tal que

$$
\left\|D f_{n_{u}}^{i}(u)-T^{i}(u)\right\|<\frac{\varepsilon}{2}, \text { e } n_{u} \geq n_{0} .
$$

Daí,

$$
\left\|D f_{n}^{i}(u)-T^{i}(u)\right\| \leq\left\|D f_{n}^{i}(u)-D f_{n_{u}}^{i}(u)\right\|+\left\|D f_{n_{u}}^{i}(u)-T^{i}(u)\right\|<\varepsilon, u \in B(1), n \geq n_{0} .
$$

Da Afirmação, mais o fato que $f_{n}(p)$ converge pontualmente, por um resultado de análise, temos que $f$ é de classe $C^{1}$, e $D f=T$. Podemos repetir este processo, indutivamente, trocando $f_{n}$ por $D f_{n}$, e assim, concluímos que $f_{n}$ converge a uma função $f \in C^{r}\left(M, \mathbb{R}^{s}\right)$. O que demonstra a proposição.

Observação A.1.3. A topologia induzida pela $\|\cdot\|_{r}$ em $C^{r}\left(M, \mathbb{R}^{s}\right)$ não depende da cobertura $V_{1}, \ldots, V_{k}$ de $M$ utilizada.

Teorema A.1.4 (Whitney). Se $M$ é uma variedade diferenciável de dimensão $m$, existe um mergulho próprio $f: M \rightarrow \mathbb{R}^{2 m+1}$. 
Observação A.1.5. Usando o Teorema A.1.4, podemos definir a topologia $C^{r}$ em $C^{r}(M, M)$. Como $M$ é compacto temos que $M \subset \mathbb{R}^{4+1}$ é um espaço métrico completo. Assim, podemos trocar $\mathbb{R}^{s}$ por $M$ na demonstração da Proposição A.1.2, e concluir que $C^{r}(M, M)$ é completo, com respeito a norma $\|\cdot\|_{r}$.

Proposição A.1.6. $\operatorname{Diff}_{\omega}^{1}(M)$ é um espaço de Baire.

Demonstração. Se mostrarmos que $\operatorname{Diff}_{\omega}^{1}(M)$ é um subespaço fechado de $C^{1}(M, M)$, pela Observação A.1.5, temos que $\operatorname{Diff}_{\omega}^{1}(M)$ também é um espaço métrico completo com respeito a norma $\|\cdot\|_{1}$. Portanto, pela Proposição A.1.1, Diff ${ }_{\omega}^{1}(M)$ é um espaço de Baire.

Mostremos, então, que Diff ${ }_{\omega}^{1}(M)$ é fechado em $C^{1}(M, M)$.

Seja $f_{n} \in \operatorname{Diff}_{\omega}^{1}(M)$ uma sequência qualquer, que converge para uma função $f \in C^{1}(M, M)$. Como $f_{n}$ preserva o elemento de área $\omega$ temos que Jac $f_{n}(x)=1$, para todo $x \in M$, e $n \in \mathbb{N}$. Disto, temos que $f$ é difeomorfismo local, com Jac $f(x)=1$, para todo $x \in M$.

\section{Afirmação 1: $f$ é sobrejetiva.}

Seja $y \in M$. Consideremos a sequência $x_{n}=f_{n}^{-1}(y)$ em $M$. Como este último é compacto, repassando $x_{n}$ para uma subsequência, se necessário, podemos assumir que $x_{n} \rightarrow x$ em $M$, quando $n \rightarrow \infty$. Como $f \in C^{1}(M, M), f\left(x_{n}\right) \rightarrow f(x)$, para $n \rightarrow \infty$. Agora, dado $\varepsilon>0$, existe $n_{0}$ tal que $\left\|f_{n}-f\right\|<\varepsilon$, para $n \geq n_{0}$. Assim,

$$
\left\|f_{n}\left(x_{n}\right)-f\left(x_{n}\right)\right\|=\left\|y-f\left(x_{n}\right)\right\|<\varepsilon, \text { para } n \geq n_{0} .
$$

E, portanto, $f\left(x_{n}\right) \rightarrow y$ para $n \rightarrow \infty$. Pela unicidade do limite, temos que $f(x)=y$. E isto conclui Afirmação 1.

\section{Afirmação 2: $f$ é injetiva.}

Suponhamos que $f(x)=f(y)$ para $x \neq y \in M$. Como $f$ é um difeomorfismo local, podemos escolher abertos $B=B(f(x), r)$, a bola aberta de centro $f(x)$ e raio $r>0, A_{1}$, e $A_{2}$ vizinhanças de $z, x$ e $y$, respectivamente; tais que $A_{1}$ e $A_{2}$ sejam disjuntos, e

$$
\begin{aligned}
& f: A_{1} \rightarrow B \\
& f: A_{2} \rightarrow B
\end{aligned} \text { sejam difeomorfismos }
$$

Definimos $\mu_{\omega}(E)=\int_{E} \omega$, para $E \subset M$. Desta forma, temos que $\mu_{\omega}$ é uma medida sobre $M$. Como Jac $f(x)=1$ para todo $x \in A_{1} \cup A_{2}$, temos que

$$
\mu_{\omega}(B)=\int_{B} \omega=\int_{A_{1}} \omega=\mu_{\omega}\left(A_{1}\right)
$$

Analogamente, $\mu_{\omega}(B)=\mu_{\omega}\left(A_{2}\right)$. Estes possuem medidas positivas pois $\omega$ é um elemento de área. Tomemos $\varepsilon=\mu_{\omega}(B)>0$, e consideremos $B^{\prime}=B(f(x), r+\delta)$ tal que $\mu_{\omega}\left(B^{\prime}\right)<3 \varepsilon / 2$.

Afirmamos que existe sequência $x_{n} \in A_{1} \cup A_{2}$ tal que $f_{n}\left(x_{n}\right) \notin B^{\prime}$. 
Como $f_{n} \in \operatorname{Diff}_{\omega}^{1}(M)$,

$$
\mu_{\omega}\left(f_{n}\left(A_{1} \cup A_{2}\right)\right)=\mu_{\omega}\left(A_{1} \cup A_{2}\right)=\mu_{\omega}\left(A_{1}\right)+\mu_{\omega}\left(A_{2}\right)=2 \mu_{\omega}(B)
$$

Suponhamos, agora, que não exista tal sequência $x_{n}$, assim $f_{n}\left(A_{1} \cup A_{2}\right) \subset B^{\prime}$, $\operatorname{logo}$

$$
\mu_{\omega}\left(B^{\prime}\right) \geq \mu_{\omega}\left(f_{n}\left(A_{1} \cup A_{2}\right)\right)=2 \mu_{\omega}(B),
$$

o que contradiz a escolha de $B^{\prime}$. E, portanto, realmente existe sequência $x_{n}$, como acima.

Repassando esta sequência $x_{n}$ para uma subsequência, se necessário, podemos assumir que $x_{n} \rightarrow z$, em $M$. Como $A_{1}$ e $A_{2}$ são fechados temos que $z \in A_{1} \cup A_{2}$, e por escolha destes conjuntos, $f(z) \in B$. Por outro lado, como $f_{n}\left(x_{n}\right) \notin B^{\prime}$, mais a escolha de $B^{\prime}$ temos que $f(z) \notin B$. Com esta contradição temos que realmente $f$ é injetiva.

Das Afirmações 1 e 2, concluímos que $f$ é um difeomorfismo, logo $f \in \operatorname{Diff}_{\omega}^{1}(M)$. E assim, $\operatorname{Diff}_{\omega}^{1}(M)$ é fechado. 


\section{Referências Bibliográficas}

[1] Newhouse, S.E. - Topological entropy and Hausdorff dimension for area preserving diffeomorphisms of surfaces, Société Mathématique de France, Astérisque, Vol. 51 (1978), 323-334 .

[2] Newhouse, S.E. - Quasi-eliptic periodic points in conservative dynamical systems, American Journal of Mathematics, Vol. 99, No. 5 (1975), 1061-1087 .

[3] Takens, F. - Homoclinic points in conservative systems, Inventiones Mathe. 18 (1972), 267-292.

[4] Smale, S.Differentiable dynamical systems, American Mathematical Society 73 (1967), 747-817 .

[5] Robinson, C. - Dynamical Systems, second edition (1998).

[6] Bowen, R. - Equilibrium states and the ergodic theory of Anosov diffeomorphisms, Lecture Notes in Mathematic, No. 470, Springer-Verlag, N.Y. (1975).

[7] Bowen, R. - Periodics Points and Measures for Axioma A Difeomorphisms, Transactions of the American Mathematical Society, Vol. 54 (1971), 377-397.

[8] Zehnder, E. - Note on smoothing symplectic and volume-preserving diffeomorphisms, Geometry and Topology, 828-854. Lectures Notes in Math., vol. 597, Springer, Berlin (1977).

[9] Mané, R. - Introdução a teoria ergódica, IMPA, CNPq, Rio de Janeiro (1983).

[10] Palis, J. and Melo, W. - Introdução aos Sistemas Dinâmicos, IMPA,CNPq, Rio de Janeiro (1978).

[11] Katok, A. and Hasselblatt, B. - Introduction to the Modern Theory of Dynamical Systems, Cambridge University Press (1995). 
[12] Hartman, P. - A Lemma in the theory of structural stability of differential equations, Proc. Amer. Math. Soc. vol 11, 610-620 (1960).

[13] Bonatti, C. and Crovisier, S. - Recurrence et généricité Preprint Dijon (2003).

[14] Munkres, J. R. - Topology, A First Course, Prentice-Hall, Inc., Englewood Cliffs, New Jersey (1975). 


\section{_Tabela de Símbolos e Abreviações}

\begin{tabular}{|c|c|}
\hline$M$ & Variedade orientável, compacta, conexa, de dimensão dois \\
\hline$\omega$ & 2-forma diferenciável, $C^{\infty}$, fechada, e não degenerada, sobre $M$ \\
\hline$f^{*} \omega$ & pull-back da forma $\omega$ por $f$ \\
\hline$C^{r}\left(M, \mathbb{R}^{s}\right)$ & $\begin{array}{l}\text { espaço das aplicações diferenciáveis de classe } C^{r}, r \geq 0 \text {, definidas sobre } \\
\text { a variedade } M \text { em } \mathbb{R}^{s}\end{array}$ \\
\hline iff $^{r}(M)$ & conjunto dos difeomorfismos de classe $C^{r}$ sobre $M, r \geq 1$. \\
\hline $\operatorname{Diff}_{\omega}^{1}(M)$ & conjunto dos difeomorfismos de classe $C^{1}$, que preservam a 2-forma $\omega$ \\
\hline $\operatorname{Jac} f(x)$ & determinante do jacobiano de $f$ \\
\hline$\tau(p)$ & período do ponto periódico $p$ \\
\hline $\operatorname{Per}(f)$ & conjunto dos pontos periódicos de $f$ \\
\hline $\operatorname{Hip}_{n}(f)$ & $\begin{array}{l}\text { conjunto dos pontos periódicos hiperbólicos de } f \text {, com período menor ou } \\
\text { igual a } n\end{array}$ \\
\hline$H(p, f)$ & conjunto dos pontos homoclínicos transversais de $p$, com respeito a $f$ \\
\hline$\lambda(p)$ & $\begin{array}{l}\text { o único autovalor de norma maior do que um, da aplicação } D f_{p}^{\tau(p)} \text {, para } \\
p \text { periódico hiperbólico }\end{array}$ \\
\hline$\Omega(f)$ & conjunto de todos os pontos não errantes de $f$. \\
\hline $\mathbb{E}^{u}(p)$ & direção instável de $f$, no ponto $p$ \\
\hline $\mathbb{E}^{s}(p)$ & direção estável de $f$, no ponto $p$ \\
\hline$W^{u}(p, f)$ & variedade instável de $p$ para $f$ \\
\hline$W^{s}(p, f)$ & variedade estável de $p$ para $f$ \\
\hline $\bar{\lambda}(p)$ & $\begin{array}{l}\text { valor absoluto do Jacobiano da aplicação } f \text {, restrita a direção instável } \\
\mathbb{E}^{u} \text {. }\end{array}$ \\
\hline$f$ & atropia topológica de $f$ \\
\hline
\end{tabular}


$\Sigma_{N} \quad$ espaço das sequências com $N$ símbolos

$\sigma($.$) \quad aplicação shift$

$H_{\mu}(\mathcal{Q}) \quad$ entropia da partição $\mathcal{Q}$ com respeito a $\mu$

$h_{\mu}(f) \quad$ entropia de $f$ com respeito a medida $\mu$

$P_{f}(\phi) \quad$ pressão topológica de $f$, com respeito ao observável $\phi$

$M_{f}(A) \quad$ conjunto das medidas de probabilidades invariantes por $f$

$S_{f} \quad$ função geradora do difeomorfismo simplético $f$

$H D(E)$ dimensão de Hausdorff de $E$

$C(z, E) \quad$ componente conexa de $E$ que contém $z$

$m(L) \quad$ conorma da aplicação linear $L$

$u(q, g) \quad$ o menor inteiro positivo $n$ tal que $m\left(D g^{n}(q) \mid \mathbb{E}_{q}^{u}\right) \geq 2$

$\mathcal{H} \quad$ subconjunto residual em $\operatorname{Diff}_{\omega}^{1}(M)$ dado pelo Teorema 3.2.5 



\section{Índice Remissivo}

aplicação

$\phi^{u}, 12$

Holder contínua, 12

shift, 9

atrator, 13

conjugação topológica, 5

conjunto

$(n, \varepsilon)$-separado, 8

básico hiperbólico, 6

hiperbólico, 4

não errante, 5

conorma , 34

continuação de um conjunto hiperbólico, 7

coordenadas simpléticas, 19

difeomorfismo

axioma $\mathrm{A}, 5$

de Anosov, 5

simplético, 15

dimensão de Hausdorff, 24

entropia

de uma partição, 11

métrica, 11

topológica, 8

estado de equilíbrio, 12

forma

diferencial exata, 16 diferencial fechada, 16

função $s(f), 24$

função geradora, 18

Lambda Lema, 6

Lema de Perturbação, 20

ponto

homoclínico, 7

periódico elíptico, 3

periódico hiperbólico, 3

periódico k-elíptico, 4

pressão topológica, 12

Princípio Variacional, 11

Teorema

da Variedade Estável, 6

de Hartman Grobman, 5

variedade simplética, 15

vizinhança

captadora, 13

isoladora, 6 